\title{
Structural and vibrational properties of deformed carbon nanotubes
}

\author{
Xiao-ping YANG $(\text { 杨晓萍 })^{1}$, Gang WU $(\text { 吴刚 })^{2}$, Jin-ming DONG $(\text { 董锦明 })^{3}$ (凶) \\ ${ }^{1}$ Max-Planck Institute für Festkörperforschung, D-70569 Stuttgart, Germany \\ ${ }^{2}$ Institute of High Performance Computing, 1 Fusionopolis Way, 16-16 Connexis, Singapore 138632, Singapore \\ ${ }^{3}$ National Laboratory of Solid State Microstructures and Department of Physics, Nanjing University, Nanjing 210093, China \\ E-mail: bunnyxp@hotmail.com,wugaxp@gmail.com,jdong@nju.edu.cn \\ Received January 25, 2009; accepted March 13, 2009
}

\begin{abstract}
The fantastic variation of the physical properties of carbon nanotubes (CNTs) and their bundles under mechanical strain and hydrostatic pressure makes them promising materials for fabricating nanoscale electromechanical coupling devices or transducers. In this paper, we review the recent progress in this field, with much emphasis on our first-principles numerical studies on the structural and vibrational properties of the deformed CNTs under uniaxial and torsional strains, and hydrostatic pressure. The nonresonant Raman spectra of the deformed CNTs are also introduced, which are calculated by the first-principles calculations and the empirical bond polarizability model.
\end{abstract}

Keywords deformed carbon nanotube, electronic structure, Raman spectra

PACS numbers 78.67.Ch, 78.30.Na, 63.22.Gh, 61.46.Fg, 73.22.-f

Contents

1 Introduction

2 Model and method

2.1 Line group theory and selection rule

2.2 Cumulant force-constant method

2.3 Empirical bond polarizability model

3 Carbon nanotube bundles under hydrostatic pressure

3.1 SWCNT bundles

3.2 DWCNT bundles

4 Raman modes of the deformed SWCNTs

4.1 Raman active modes of the SWCNTs under a uniaxial tensile strain

4.2 Raman active modes of the SWCNTs under a torsional strain

4.3 Radial-breathing-like mode of the collapsed SWCNT bundles under a hydrostatic pressure

5 Conclusions

Acknowledgements

References

\section{Introduction}

In the past decade, carbon nanotubes (CNTs) $[1-3]$ had been extensively investigated due to their special electri- cal and mechanical properties, as well as their potential applications in future nano-structured materials, such as nano-scale quantum wires, single electron and field-effect transistors and sensors.

The single-walled carbon nanotube (SWCNT) is a hollow cylinder rolled up from graphite sheet with its diameter of a few to tens of angstroms, and length of several microns to several millimeters. Its geometrical structure can be described by a pair of integers $(n, m)$, which determines its radius and chirality, and so entirely its electronic structure, and optical property. It is known that the SWCNTs with $n-m$ being a multiple of 3 are metallic, and all others are semiconducting [4].

Since its discovery [1] in 1991 by Iijima, the SWCNT has been of great interest in nano-scale physics and nanotechnology because of its exceptional electronic and mechanical properties [5]. Due to its characteristic onedimensional (1-D) geometric structure, the SWCNT's electron and phonon wave vector are quantized in its circumferential direction and quasi-continuous along its tube axis. Also, the Van Hove singularities (VHSs) appear in both electronic and phonon densities of states (DOS). The SWCNT's electronic properties can vary significantly, depending sensitively on their diameters and chiral angles. At the same time, their vibrational properties also show special behaviors, such as radial breathing 
mode $(\mathrm{RBM})$, rotational mode, and 1-D phonon dispersion curves. As a consequence, the Raman spectra of the SWCNTs show many new phenomena, which are different from those of other 3-D materials.

In the recent decades, much attention has been devoted to the vibrational properties of the SWCNTs $[6-20]$. The low-frequency peaks in their Raman spectra are attributed to the RBMs ( $R$ band), where all carbon atoms are subject to an in-phase radial displacement. It is found that the RBM frequency depends only on the tube's diameter in an inverse proportion, i.e.,

$$
f_{\mathrm{RBM}}=D / d_{t}
$$

where $D$ is a constant and $d_{t}$ is the tube's diameter. On the other hand, the higher frequency peaks in their Raman spectra are caused by the out-of-phase vibrations of the neighboring carbon atoms parallel to the tube's surface (tangential modes, $T$ band), which are related to the $E_{2 g}(2)$ phonon mode of $\sim 1580 \mathrm{~cm}^{-1}$ in the graphite. In the achiral tubes, one can distinguish two tangential modes with atomic displacements parallel or perpendicular to the tube axis. More detailed discussions about the general features of the SWCNT's vibrational modes can be found in Ref. [4].

There exists a strong electron-phonon coupling in the SWCNTs, which greatly enhances their resonant Raman scattering, and further induces the various Stokes and anti-Stokes peaks in their Raman spectra. As a result, the Raman spectrum has become one of the dominant experimental techniques to detect the diameter-selective phonon modes and to study the vibrational properties of the CNTs [7, 21-23]. It is also expected that one can use Raman spectra to characterize the detailed structural information of the CNT-based materials, such as the SWCNTs, the double-walled CNTs (DWCNTs) and their bundles.

On the other hand, the DWCNT is the simplest type of multi-walled CNT (MWCNT), which has been made experimentally by many different methods. The nucleation of its inner tube should occur after the growth of the outer tube according to the "yarmulke mechanism" [24], implying the inner tube diameter is determined by that of the outer tube. A DWCNT can be formed by a pair of inner and outer constituent layers with any chirality, leading to different kinds of DWCNTs, such as metal-metal, metal-semiconductor, or semiconductorsemiconductor DWCNTs. Early studies on the DWCNT focused mainly on their stability and electronic structures, etc. [25-28]. It was shown that the band structure of a DWCNT depends on the combined configurations of its inner and outer tubes [26], but its stability depends only on its interlayer spacing [25].

The CNTs are usually supported on a solid substrate in the experiments, causing various mechanical deformations of the CNTs, which lead to corresponding changes of their electronic structures, demonstrated by the atomic force microscopy and molecular dynamics calculations $[29,30]$. For example, the symmetry breaking due to the deformation may remove their energy level degeneracy. And an increased curvature caused by the deformation can enhance the $\sigma-\pi$ mixing and rehybridization. Thus, there have been a lot of experimental and theoretical researches on the electronic properties of the deformed CNTs because of their diverse possible applications, e.g., electromechanical actuators [31].

The resistance of an SWCNT transistor was found experimentally to vary significantly under the tube's bending and stretching [32], and an observed torsion of a metallic SWCNT was speculated to open a small band gap [33]. On the other hand, several theoretical studies had been made on the electronic properties of the deformed SWCNTs under two kinds of strains, i.e., the uniaxial and torsional [34-39], which had shown that the band structures of the deformed SWCNTs are determined by their chiral symmetries and the kind of strains. It was also found that most of the deformed SWCNTs show a metal-semiconductor transition, occurring periodically with the increasing strain. Under a uniaxial strain, the derivative of the band gap over strain is the largest for the zigzag tubes, decreasing with the increase of the chiral angle. In contrast, under a torsional strain, the derivative becomes the largest for the armchair tubes, decreasing as the chiral angle decreases. In particular, the armchair tubes under a uniaxial strain and the metallic zigzag tubes under a torsional strain remain metallic $[37,38]$.

It is well known that the physical properties of the CNTs depend much on their geometrical structures, and so can be easily changed by an applied pressure or strain, which could be used to fabricate the nanoscale electromechanical coupling devices and transducers. The aim of this article is to summarize recent advances in the investigation of the structural, electronic, optical and vibrational properties of the CNTs and CNT bundles under mechanical deformation and hydrostatic pressure, paying attention mainly on our researches on them.

This paper is organized as follows. In Section 2, we first review the fundamental theoretical methods to calculate the phonon dispersions and the Raman spectra of the CNTs, which are the line group theory, the Cumulant force-constant (CFC) method and the empirical bond polarizability model (EBPM) to calculate the nonresonant Raman spectra. They actually form the theoretical foundations of our researches. Then, in Section 3, the structural phase transitions (SPT) of both SWCNT and DWCNT bundles under a hydrostatic pressure have been investigated. And in Section 4, the nonresonant Raman spectra of the chiral and achiral SWCNTs under tensile 
and torsional strains have been systematically studied by a combination of the first principles calculations and the EBPM. In the same section, the vibrational properties and nonresonant Raman spectra of $(10,10)$ SWCNT bundle under hydrostatic pressure are studied too, and specifically, a radial-breathing-like mode has been found for the SWCNT bundle after the SPT. Finally, a conclusion is given in Section 5.

\section{Model and method}

\subsection{Line group theory and selection rule}

The SWCNT can be formed by wrapping a graphene sheet into a seamless cylinder, for which, however, there are infinite possibilities to do so, and each wrapping causes an SWCNT with a pair of specific tube radius and chiral angle. Therefore, a correct symmetric analysis is the only way to reveal the underlying relationship among the infinite possible tubes. Especially, the phonon vibrational properties have a closed relation with the system symmetry, and the selection rules of the infrared (IR) and Raman spectra can also be derived from the group theory. In addition, introducing the symmetric analysis has another important effect on the realistic calculations, which could reduce the overall computational requirements and improve the accuracy.

The most advanced group theory for the SWCNTs is the line group theory suggested by Damnjanović et al. [40-44]. By introducing generalized translational operations, it is found that the full symmetries of infinitely long SWCNTs form the non-Abelian nonsymorphic line groups [45], enlarging the groups reported in the literature [4]. This fact will directly affect the number of the SWCNT's IR and Raman active modes [46]. Here, we cannot say too much about the line group theory, and interested readers can refer to Refs. [40-44]. Instead, here we will collect only the main results about the selection rule.

Because the nonresonant Raman spectrum is related only to the phonons at the $\Gamma(k=0)$-point, we would like to consider the irreducible representations of the symmetric group at the $\Gamma$ point.

It has been shown [46] that the irreducible representations of the SWCNT's Raman active modes are given by

$$
\begin{aligned}
& \Gamma_{\text {Raman }}^{\text {zigzag }}=2 A_{1 g} \oplus 3 E_{1 g} \oplus 3 E_{2 g} \\
& \Gamma_{\text {Raman }}^{\text {armchair }}=2 A_{1 g} \oplus 2 E_{1 g} \oplus 4 E_{2 g} \\
& \Gamma_{\text {Raman }}^{\text {chiral }}=3 A_{1 g} \oplus 5 E_{1} \oplus 6 E_{2}
\end{aligned}
$$

Thus, the number of Raman active modes in different kinds of tubes are $n_{\text {Raman }}^{\text {zigzag }}=2+3+3=8, n_{\text {Raman }}^{\text {armchair }}=$ $2+2+4=8$, and $n_{\text {Raman }}^{\text {chiral }}=3+5+6=14$. That's to say, the number of Raman active modes in the achiral tubes is only about half of that in the chiral tubes due to their higher symmetry. It can be expected that the number of Raman active modes in achiral tubes will increase if the tube's symmetry is broken, implying also the appearance of new Raman active modes. Therefore, it is possible to use these Raman modes to detect the deformation type or even the intensity of the deformation in the SWCNTs.

\subsection{Cumulant force-constant method}

In solid state physics, the phonon dispersions can be determined by the secular equation:

$$
\operatorname{det}\left|\frac{1}{\sqrt{M_{s} M_{t}}} D_{s t}^{\alpha \beta}(\boldsymbol{q})-\omega^{2}(\boldsymbol{q})\right|=0
$$

where, subscripts of $s$ and $t$ indicate the atomic index in the unit cell. $\alpha$ and $\beta$ denote the $x, y$ or $z$ directions, and $\boldsymbol{q}$ is the phonon wave vector. $M_{s}$ is the mass of the $s$-th atom. $\omega$ is the vibrational frequency. Dynamic matrix $D_{s t}^{\alpha \beta}(\boldsymbol{q})$ is defined by

$$
D_{s t}^{\alpha \beta}(\boldsymbol{q}) \equiv \sum_{\mathbf{R}} \mathrm{e}^{-\mathrm{i} \boldsymbol{q} \cdot \boldsymbol{R}} C_{s t}^{\alpha \beta}(\boldsymbol{R})
$$

where $C_{s t}^{\alpha \beta}(\boldsymbol{R}) \equiv \frac{\partial^{2} E}{\partial u_{s}^{\alpha}(l) \partial u_{t}^{\beta}(m)}$ is the force constant. $E$ is the total energy, and $\boldsymbol{R} \equiv \boldsymbol{R}_{l}-\boldsymbol{R}_{m}$ is the distance between the $l$-th and the $m$-th unit cell in the Bravais lattice. $u_{s}^{\alpha}(l)$ stands for the displacement of the $s$-th atom in $l$-th unit cell along the $\alpha$ direction.

In most of the published papers on the SWCNT's phonon dispersions, using ab initio calculations, the low-frequency dispersions were not well obtained due mainly to the use of the interatomic force-constant (IFC) method. The reason is that the summation in Eq. (4) is not really performed over all $\boldsymbol{R}$ in the IFC method. In many cases, the $\boldsymbol{R}$ must be cut at a modest distance. Such a treatment makes the frequency of the acoustic phonon at $\Gamma$ point to be not exactly zero.

Ye et al. [47] successfully calculated phonon dispersions of the SWCNTs and graphite by means of the $\mathrm{Cu}$ mulant Force Constant (CFC) method [48]. The details about a comparison between the CFC and IFC methods are available in their original paper [47]. This is exactly the method to be used in this work to calculate the SWCNT's phonon dispersion. So, it is valuable to summarize the advantage of employing the CFC method to calculate the phonon dispersions of the CNTs.

(a) The 1D-like geometrical structure of the carbon nanotube makes its physical properties highly anisotropic, for which it is definitely necessary to use the CFC method instead of the IFC one to study its phonon dispersions. Although the CFC method is still an approximated one, it keeps both the translational and rotational symmetries. As a result, the symmetries of 
the dynamical matrices are correct in spite of the fact that the dynamical matrices are obtained approximately. Therefore, all the vibrational properties can be reproduced correctly by the CFC method. This is the reason why it is important for an anisotropic system such as the carbon nanotube.

(b) For the IFC method, one has to take a supercell large enough to minimize the truncation effect. It is very computationally time-consuming. At the same time, the numerical noise in force calculations may become very serious if a too large supercell is used, which may reduce the calculation accuracy. On the other hand, because the CFC method does not need to perform an explicit truncation on the interatomic interactions, as shown in Ref. [47], only a very small supercell $(1 \times 1 \times 2)$ is sufficient for the CFC method to calculate accurately the phonon modes on the $\Gamma$ and $X$ points at Brillouin zone boundary. This fact is most important for the chiral SWCNTs. For example, the $(12,4)$ tube contains 208 atoms in its unit cell. With the CFC method, we can also afford the phonon dispersion calculations under a limited hardware condition.

\subsection{Empirical bond polarizability model}

The time-averaged power flux of the Raman-scattered light at a given direction of a solid angle $\mathrm{d} \Omega$ in a frequency range of $\omega_{f}$ and $\omega_{f}+\mathrm{d} \omega_{f}$ is related to the differential scattering cross section as follows:

$$
\begin{aligned}
\frac{\mathrm{d}^{2} \sigma}{\mathrm{d} \Omega d \omega_{f}}= & \frac{\omega_{f}^{3} \omega_{i} \hbar}{8 \pi^{2} c^{2}}[\langle f(\omega)\rangle+1] \\
& \cdot \sum_{\alpha \beta \gamma \lambda} v_{\alpha} v_{\beta} H_{\alpha \gamma \beta \lambda}(\omega) w_{\gamma} w_{\lambda}
\end{aligned}
$$

where

$$
\begin{aligned}
H_{\alpha \gamma \beta \lambda}(\omega)= & \sum_{j} a_{\alpha \gamma}^{*}(j) a_{\beta \lambda}(j) \\
& \cdot \frac{1}{2 \omega_{j}}\left[\delta\left(\omega-\omega_{j}\right)+\delta\left(\omega+\omega_{j}\right)\right]
\end{aligned}
$$

with

$$
a_{\alpha \gamma}(j)=\sum_{n \delta} \frac{\pi_{\alpha \gamma, \delta}^{n}}{\sqrt{M_{n}}}\langle n \delta \mid j\rangle
$$

Here, $\omega_{i}$ and $\omega_{f}$ are the frequencies of the incident and scattered light; $\omega \equiv \omega_{i}-\omega_{f}$ is the Raman shift. $\boldsymbol{v}$ and $\boldsymbol{\omega}$ denote the corresponding polarization unit vectors of the incident and scattered light, respectively. $\langle f(\omega)\rangle$ is the Bose factor. $n$ is the atomic index and $M_{n}$ is the mass of the $n$-th atom. $\alpha, \beta, \gamma$, and $\lambda$ indicate the $x, y$, or $z$ component of the vector. $\omega_{j}$ and $\langle n \delta \mid j\rangle$ are the frequency and $n \delta$-component of the $j$-th mode. The coefficient $\pi_{\alpha \gamma, \delta}^{n}$ in Eq. (7) connects the polarization changes to the atomic motions [49], which is obtained by expanding the polarizability tensor $\pi^{n}$ in terms of the atom displacements $u_{\delta}^{n}$, with

$$
\pi_{\alpha \gamma, \delta}^{n}=\sum_{m}\left(\frac{\partial \pi_{\alpha \gamma}^{m}}{\partial u_{\delta}^{n}}\right)_{0}
$$

Although it has been shown that the polarizability coefficient $\pi_{\alpha \gamma, \delta}^{n}$ can be calculated within the density functional theory [50], the empirical bond-polarization model (EPBM) [11, 51, 52] still offers an efficient way to calculate the nonresonant Raman spectra of carbon nanotubes. In the EBPM, the Eq. (8) can be rewritten as:

$$
\begin{aligned}
\pi_{\alpha \beta, \gamma}^{n}= & \sum_{m} \frac{\left(2 \alpha_{/ /}^{\prime}+\alpha_{\perp}^{\prime}\right)}{3} \delta_{\alpha \beta} \hat{r}_{\gamma} \\
& +\left(\alpha_{/ /}^{\prime}-\alpha_{\perp}^{\prime}\right)\left(\hat{r}_{\alpha} \hat{r}_{\beta}-\frac{1}{3} \delta_{\alpha \beta}\right) \hat{r}_{\gamma} \\
& +\frac{\left(\alpha / /-\alpha_{\perp}\right)}{r}\left(\delta_{\alpha \gamma} \hat{r}_{\beta}+\delta_{\beta \gamma} \hat{r}_{\alpha}-2 \hat{r}_{\alpha} \hat{r}_{\beta} \hat{r}_{\gamma}\right)
\end{aligned}
$$

Here, $\hat{r}$ is the unit vector of the $\boldsymbol{r}$ connecting the $n$ and $m$ atoms linked by a bond. $\alpha_{/ /}$and $\alpha_{\perp}$ represent the static longitudinal and perpendicular bond polarizability, respectively, and $\alpha_{/ /}^{\prime}=\left(\frac{\partial \alpha / /}{\partial r}\right)_{0}$, $\alpha_{\perp}^{\prime}=\left(\frac{\partial \alpha_{\perp}}{\partial r}\right)_{0}$. The values of $\alpha_{/ /}, \alpha_{\perp}, \alpha_{/ /}^{\prime}$ and $\alpha_{\perp}^{\prime}$ are given empirically as a function of the bond length between two carbon atoms. For undeformed SWCNTs, there is only one type of bonds and the bond polarizability model is completely defined by three parameters [11]: $\quad \alpha=2 \alpha_{/ /}^{\prime}+\alpha_{\perp}^{\prime}=4.7 \AA^{2}$, $\beta=\alpha_{/ /}^{\prime}-\alpha_{\perp}^{\prime}=4.0 \AA^{2}$, and $\gamma=\alpha_{/ /}-\alpha_{\perp}=0.04 \AA^{3}$. It is known, however, that the polarizability parameters of carbon are similar for a variety of carbon materials. Furthermore, the relative intensities of the Raman modes are not so sensitive to small changes of the bond polarization parameter values, except for the lowest $E_{2 g}$ mode. Thus, it is a reasonable approximation to keep the above polarization parameters for the deformed SWCNTs. This approximation leads to a small error in the peak height, but does not affect the peak positions. In fact, Guha et al. [52] chose different bond polarizability parameters to calculate the fullerene's Raman spectra, finding only small changes of the relative Raman intensities.

In the following text, the line shape of each peak is assumed to be Lorentzian and its line-width is fixed at $1.68 \mathrm{~cm}^{-1}$ by default. The tube axis is taken along the $Z$ direction, and the $X$ and $Y$ directions are taken to be perpendicular to it. Generally, there are two kinds of special orientations for the light polarization [11]: the $V V$ and $V H$ configurations. In the $V V$ configuration, the incident and the scattered light polarizations are parallel to each other, while they are perpendicular to each other in the $V H$ configuration. Because the intensity of the Raman peak is of less interest than its position, which is determined by the frequency of corresponding phonon mode rather than the polarization's orientation, only the Raman spectra in the $V V$ configuration are presented by 
default.

\section{Carbon nanotube bundles under hydro- static pressure}

\subsection{SWCNT bundles}

Recently, the effect of hydrostatic pressure on the CNT bundle has attracted much attention in experiments [53-61], and theoretical calculations [62-71]. It is found that the Raman peaks of the SWCNT bundles shift to higher frequencies with increasing hydrostatic pressure, and the radial breathing mode (RBM) disappears from the spectrum above the critical pressure, showing a structural phase transition (SPT) at a critical pressure. Elliott et al. [64] reported their classical molecular dynamics simulations on the SWCNT bundles under hydrostatic pressure, finding the bundle can collapse to a herringbone structure after the SPT, and their Raman spectrum measurement showed an excellent agreement with their simulations. However, Zhang et al. [65-67] put forward that the stress-induced herringbone structure is not the most favorable in energy, but found a parallel array of the collapsed nanotubes, which is even more favorable. Yang et al. [69] found that an in-between configuration under the collapse pressure (see Fig. 1), which is distinct from both the parallel and herringbone structures found previously, but is almost the degenerate in enthalpy with
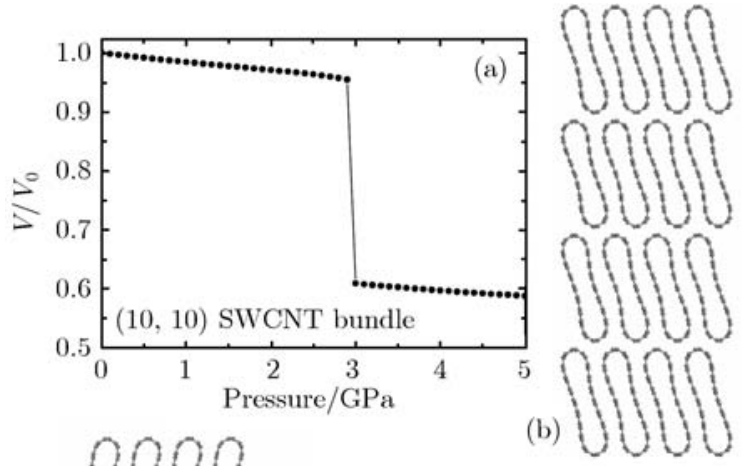

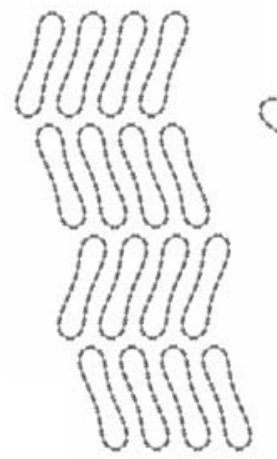

(c)

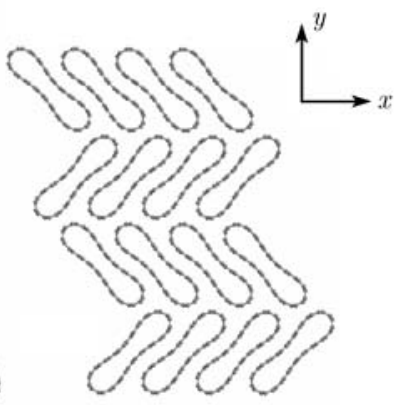

(d)
Fig. 1 (a) Loading curve for (10, 10) SWCNT bundle as a function of hydrostatic pressure. Snapshots of the cross section of a (10, 10) SWCNT bundle at 3 GPa: (b) The parallel structure, (c) The in-between structure, and (d) The herringbone structure. Adapted from Ref. [69] with permission. the parallel structure. The largest difference of the enthalpy per atom in the three structures is only about $2.4 \mathrm{meV}$.

The electronic band-structure along the tube's symmetry axis $\Gamma X$, for an individual $(10,10) \mathrm{SWCNT}$ and its bundle at $0 \mathrm{GPa}$, and the three different collapsed structures at $3 \mathrm{GPa}$ are calculated by the density functional theory (DFT) in local density approximation (LDA), and obtained results are given in Fig. 2(a)-(e), respectively [69]. The intertube van der Waals forces and the structure deformation break the rotational symmetry of the SWCNT even at zero pressure, making its energy bands near Fermi Level split, as shown in Fig. 2(b), which is different from that of an individual SWCNT [Fig. 2(a)] at the same zero pressure. Although the three collapsed structures always remain to be metallic, there still exists a basic difference between their band structures. For example, the band structure along the $\Gamma X$ direction [Fig. 2(c)] of the parallel structure shows a pseudogap of $0.2 \mathrm{eV}$ at the Fermi Level, and another small one of $0.08 \mathrm{eV}$ emerges in the electronic band of the herringbone structure [Fig. 2(e)]. However, no pseudo-gap exists in the band of the in-between structure, in which the top of the valence band and the bottom of the conduction band lie separately at different $k$ points without the pseudo-gap in Fig. 2(d). This kind of band differences between three collapsed structures comes from the different weak dispersions along the directions perpendicular to the tube axis, caused by the different numbers and relative orientation of the inequivalent tubes in their unit cells.

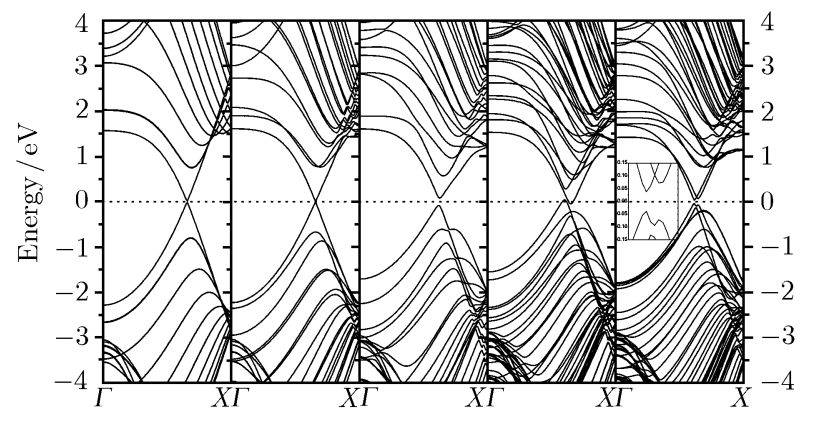

(a)

(b)

(c)

(d)

(e)

Fig. 2 The calculated electronic band structures along the nanotube symmetry axis $\Gamma X$ of (a) an individual $(10,10)$ SWCNT at $0 \mathrm{GPa}$, (b) a $(10,10)$ SWCNT bundle at $0 \mathrm{GPa}$, and of three collapsed structures at $3 \mathrm{GPa}$ : (c) The parallel structure, (d) The in-between structure, (e) The herringbone structure. Inset of (e) shows the pseudo-gap at the Fermi level in an energy region of -0.15 to $0.15 \mathrm{eV}$. The Fermi level is set at zero. Adapted from Ref. [69] with permission.

The above changes of the electronic structures in the three collapsed structures can be well reflected in their optical absorptions, given in Fig. 3. It is known from Fig. 3(a) that the individual $(10,10)$ SWCNT has six absorption peaks polarized along the tube direction $(Z$ direction). However, for the bundle at zero pressure, the 
original characteristic peaks of the individual SWCNT are slightly shifted due to the band splitting caused by the tube-tube interaction in the bundle, as seen clearly in Fig. 3(b). on the other hand, a strong absorption peak labeled "0" polarized along tube direction emerges due to the existence of the pseudo-gap along the direction parallel to the $\Gamma X$, which had been predicted by Delaney et al. $[72,73]$ and further confirmed by a later experiment [74].

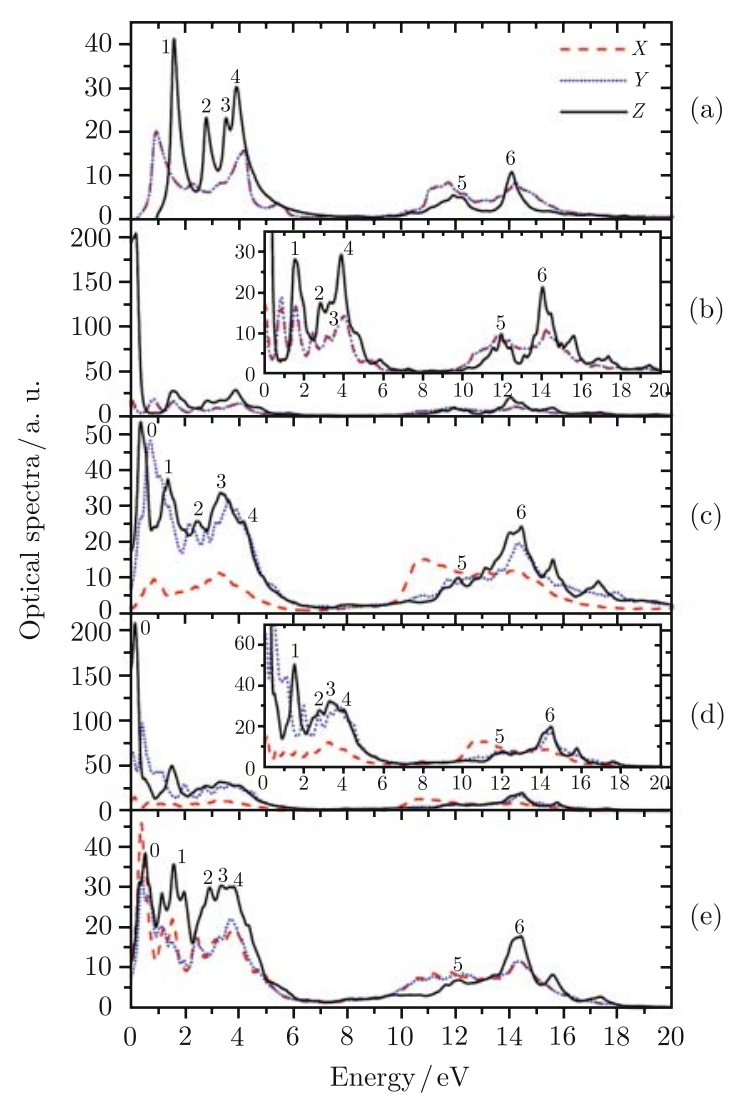

Fig. 3 The calculated imaginary part (absorptive part) of dielectric function polarized along $X, Y$ and $Z$ directions, respectively, for (a) an individual $(10,10)$ SWCNT at $0 \mathrm{GPa}$, (b) a $(10,10)$ SWCNT bundle at $0 \mathrm{GPa}$, and for three collapsed structures at 3 GPa: (c) The parallel structure, (d) The in-between structure, and (e) The herringbone structure. Adapted from Ref. [69] with permission.

It is seen clearly from Fig. 3 that there exist larger differences among the optical absorptions of the three different collapsed structures, especially in the lower frequency regions, which could be used to identify the different structures experimentally.

On the other hand, it can be found from Fig. 3(b)-(e) that there exist the large differences in the optical absorption anisotropy for the undeformed $(10,10)$ SWCNT bundle and other three collapsed configurations, which are listed as follows: (1) First of all, the optical responses polarized along the $X$ and $Y$ directions are almost the same in magnitude in the undeformed and the herringbone structures, but much different in the parallel and the in-between ones, which could be caused by the relative orientations between the nearest tubes in them. (2) In addition, it can be found that the optical responses along the $X$ direction for both of the parallel and inbetween ones are almost the same in magnitude too, but weaker than that polarized along the $Y$ direction in the low-energy region, because in both structures the normal of the flat segment of the collapsed tubes makes an almost the same angle with $X$ or $Y$ directions. The flat segment of the collapsed dumbbell-like cross section [see Fig. 1(b)] looks very similar to the graphite sheet. It is well known that in graphite, the optical absorption component polarized perpendicular to the carbon-layer is much smaller than that parallel to the layer [75]. It is seen clearly from Fig. 1(b) and (c) that the $Y$ component of the optical absorption in both parallel and in-between structures has a more parallel component of the graphite than the $X$ component, which is finally reflected in Fig. 3(c) and (d). The repeated calculations for other SWCNT bundles with the larger radius [for example, the armchair $(12,12),(14,14),(16,16),(18,18)$ and $(20,20)$ tubes] suggested that the flat segments in their cross sections become the larger and larger with increasing tube radius, indicating the graphite-like characteristic anisotropy would be more notable in the collapsed SWCNT bundles with larger radii. However, this character is absent in the optical spectra of the herringbone configuration because as seen from Fig. 1(d), its $X$ and $Y$ directions form an almost the same angle with the flat segment of the collapsed tubes, so that the $X$ and $Y$ components of its optical absorptions contain almost the same amount of the parallel component of the graphite.

As for the vibrational properties, the RBM is the most important low-frequency mode in the Raman spectra of a perfect SWCNT, in which all carbon atoms are subject to an in-phase radial displacement. It was found that the RBM frequency of an SWCNT is independent of its chiral angle and depends only on its diameter in an inverse proportion. Theoretical nonresonant Raman spectra of a $(10,10)$ SWCNT bundle at $0 \mathrm{GPa}$ and its three different collapsed structures at $3 \mathrm{GPa}$ are shown in Fig. 4. From Fig. 4(a) and its inset, it can be found that the RBM of the individual SWCNT moves from $177 \mathrm{~cm}^{-1}$ to the higher energy $205 \mathrm{~cm}^{-1}$ in the undeformed bundle due to the van der Waals forces between tubes. Meantime, its low-frequency peak at about $100 \mathrm{~cm}^{-1}$ seen in the inset of Fig. 4(a) splits, producing several peaks in Fig. 4(b). After the SPT, it is clearly seen from Fig. 4(b)-(d) that the Raman spectra of three collapsed structures have been changed greatly: (1) the whole Raman spectra extend more widely than before the SPT; (2) more Raman peaks emerge, e.g., the big distinctive peak at about 300 $\mathrm{cm}^{-1}$; (3) Most importantly, the Raman peaks shift to higher frequencies. In the Raman frequency range from 
180 to $350 \mathrm{~cm}^{-1}$, the detailed analysis on the vibrational modes of the Raman peaks was done, but no RBM-like mode is found, i.e., the original RBM of the undeformed structure disappears from this frequency region, which is well consistent with the experimental results $[56,57]$.

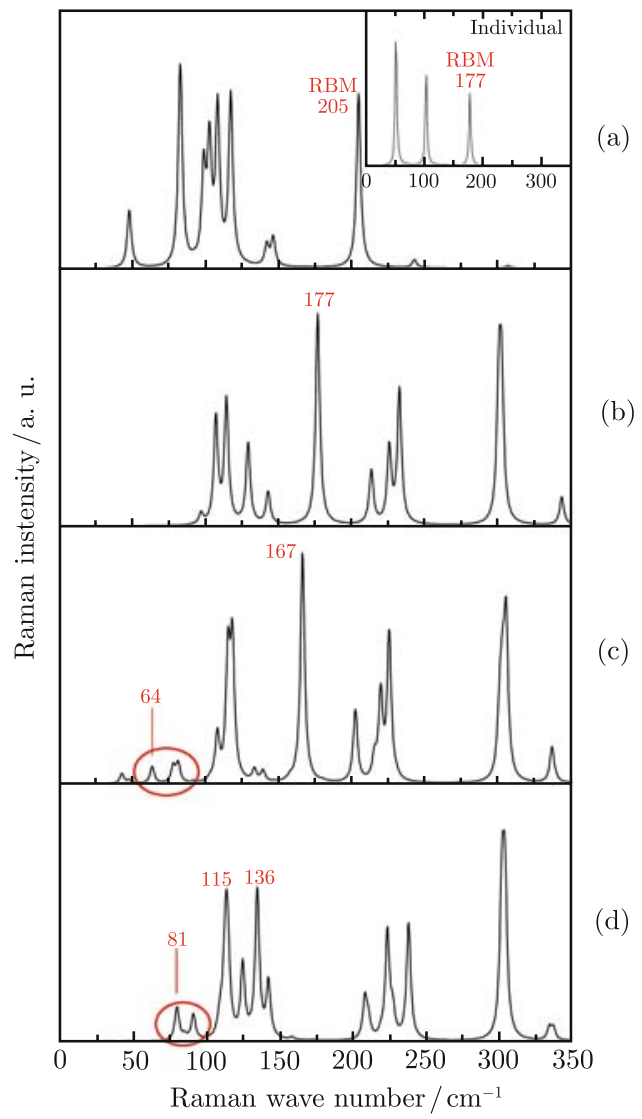

Fig. 4 The calculated nonresonant Raman spectra of (a) a (10, 10) SWCNT bundle at $0 \mathrm{GPa}$ (its inset shows that of an individual $(10,10)$ SWCNT), and of three collapsed structures at $3 \mathrm{GPa}$ : (b) The parallel structure, (c) The in-between structure, and (d) The herringbone structure. Adapted from Ref. [69] with permission.

All these changes in the Raman spectra are caused by the large differences between their structures before and after the SPT, leading to much different normal vibrational modes after the SPT. Specifically, the vibrational mode analysis revealed that there are two main differences in them: first, a distinct Raman peak at 177 $\mathrm{cm}^{-1}$ (quadrupole vibration mode) in the parallel structure disappears from the corresponding spectrum range of the herringbone structure, in which, however, the quadrupole vibrations induce two strong Raman-active peaks at 115 and $136 \mathrm{~cm}^{-1}$ due to coupling with other vibration modes in low-energy region. Second, some Raman peaks appear below $100 \mathrm{~cm}^{-1}$ in the herringbone structure, which should be mainly produced by the relative vibrations between two inequivalent tubes in its unit cell. Therefore, these results indicate that the three collapsed structures can also be distinguished clearly by their low frequency Raman-active modes.

\subsection{DWCNT bundles}

Up to now, there are only a few studies of the DWCNT bundle under the hydrostatic pressure, among which the Raman measurements [53-55] have found that the RBM intensity of the outer tubes decreases rapidly with increasing pressure, exhibiting a similar behavior to that of the SWCNT bundles [56-67], in contrast with the considerably less sensitivity to the pressure for the inner (e.g., the pressure coefficient of the inner tube is $45 \%$ smaller than that of the outer tube.).

It is found by numerical simulation using the universal force field (UFF) method [70] that there exist three kinds of the response behaviors to external pressure for different DWCNTs. Their loading curves vs. hydrostatic pressure are given in Fig. 5(a)-(c), respectively. The two transition pressures, $P_{c}$ and $P_{d}$, for different DWCNT bundles are listed in Table 1.

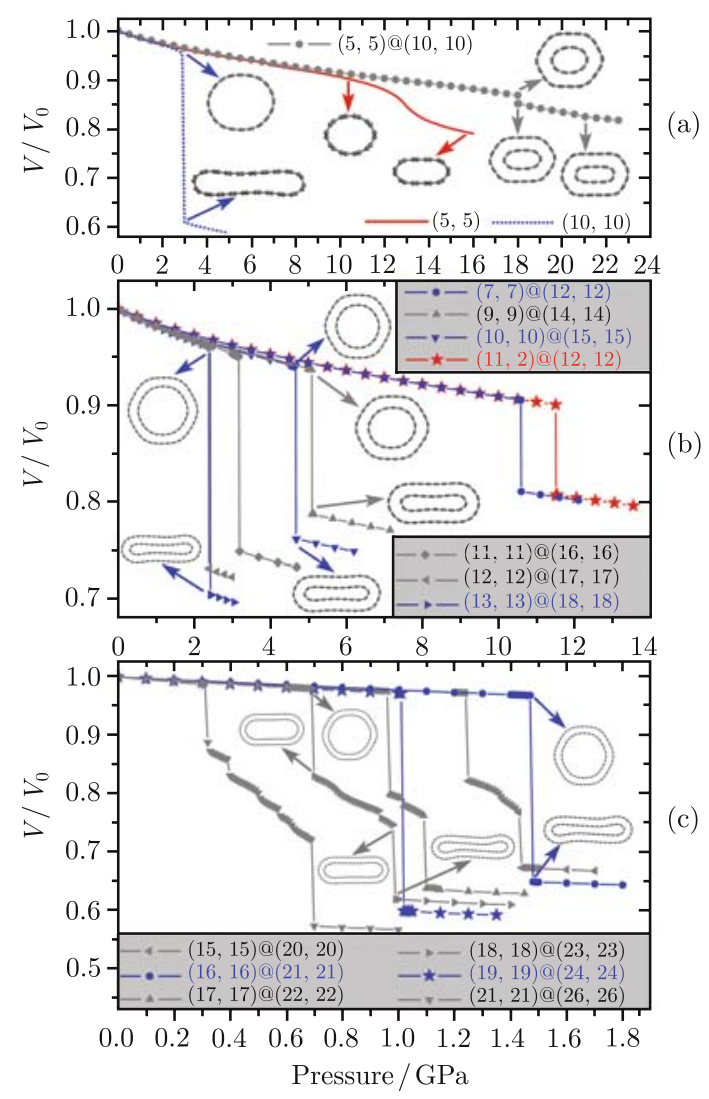

Fig. 5 Loading curves for different armchair@armchair DWCNT bundles as a function of hydrostatic pressure. (a) Small-diameter $(5,5) @(10,10)$ DWCNT bundle; (b) Some intermediate-diameter DWCNT bundles; (c) The larger-diameter DWCNT bundles. Adapted from Ref. [70] with permission.

First, for small-diameter $(5,5) @(10,10)$ DWCNT bundle, a small discontinuous volume change appears at $P=18.01 \mathrm{GPa}$, accompanied by a cross section's change between two deformed hexagons, as seen from Fig. 5(a). At the same time, the inter tube $(5,5)$ could not collapse with a minimum distance of $4.35 \AA$ between its two op- 
Table 1 Calculated critical transition pressure $P_{c}$ and $P_{d}$ of DWCNT bundles. $R_{\text {ave }}$ is the average value of the inner and outer tube's radius in the isolate DWCNT directly rolled up from the ideal graphene sheet. The suffix (NC) means no collapse happens at this pressure. Adapted from Ref. [70] with permission.

\begin{tabular}{|c|c|c|c|}
\hline DWCNT bundle & $R_{\text {ave }}$ & $P_{c}$ & $P_{d}$ \\
\hline$(5,5) @(10,10)$ & 5.085 & & $18.01(\mathrm{NC})$ \\
\hline$(7,7) @(12,12)$ & 6.441 & & 10.6 \\
\hline$(11,2) @(12,12)$ & 6.441 & & 11.51 \\
\hline$(9,9) @(14,14)$ & 7.797 & & 5.1 \\
\hline$(10,10) @(15,15)$ & 8.475 & & 4.68 \\
\hline$(11,11) @(16,16)$ & 9.153 & & 3.19 \\
\hline$(12,12) @(17,17)$ & 9.831 & & 2.39 \\
\hline$(13,13) @(18,18)$ & 10.509 & & 2.43 \\
\hline$(15,15) @(20,20)$ & 11.865 & 1.25 & 1.44 \\
\hline$(16,16) @(21,21)$ & 12.543 & & 1.48 \\
\hline$(17,17) @(22,22)$ & 13.221 & 0.97 & 1.1 \\
\hline$(18,18) @(23,23)$ & 13.899 & 0.7 & 0.99 \\
\hline$(19,19) @(24,24)$ & 14.577 & & 1.02 \\
\hline$(21,21) @(26,26)$ & 15.933 & 0.32 & 0.7 \\
\hline$(7,0) @(16,0)$ & 4.502 & & $30.5(\mathrm{NC})$ \\
\hline$(9,0) @(18,0)$ & 5.284 & & 20.24 \\
\hline$(11,0) @(20,0)$ & 6.067 & & 12.12 \\
\hline$(13,0) @(22,0)$ & 6.85 & & 7.69 \\
\hline$(15,0) @(24,0)$ & 7.633 & & 6.61 \\
\hline$(17,0) @(26,0)$ & 8.416 & & 4.42 \\
\hline$(19,0) @(28,0)$ & 9.199 & & 2.99 \\
\hline$(21,0) @(30,0)$ & 9.982 & & 3.61 \\
\hline$(23,0) @(32,0)$ & 10.765 & & 1.82 \\
\hline$(25,0) @(34,0)$ & 11.548 & 1.52 & 1.55 \\
\hline$(27,0) @(36,0)$ & 12.331 & & 2.23 \\
\hline$(29,0) @(38,0)$ & 13.113 & 0.85 & 1.13 \\
\hline$(31,0) @(40,0)$ & 13.896 & 0.71 & 0.99 \\
\hline$(33,0) @(42,0)$ & 14.679 & & 1.01 \\
\hline
\end{tabular}

posite walls, which is larger than the inter-layer distance of $3.4 \AA$ in the turbostratic graphite. If the pressure is increased further, this distance will approach continuously to $3.4 \AA$. On the other hand, simulation indicates that the loading curve of $(5,5)$ SWCNT bundle [also shown in Fig. 5(a)] varied with external pressure is continuous too and no obvious SPT happens. However, the (10, 10) SWCNT bundle collapses at $P_{d}=3 \mathrm{GPa}$, forming a peanut-shaped cross section with a separation of about $3.4 \AA$ between its two opposite parallel walls. Therefore, it is clear that the existence of an inner $(5,5)$ tube increases the ability of the outer $(10,10)$ tube to resist the applied pressure, so that the $(5,5) @(10,10)$ DWCNT bundle does not collapse at 18.01 GPa.

As for the intermediate-diameter DWCNT bundles, e.g., the $(7,7) @(12,12),(9,9) @(14,14),(10,10) @(15$, 15), (11, 11)@(16, 16), (12, 12)@(17, 17) and (13, $13) @(18,18)$, as shown in Fig. 5(b), it is found that all of them undergo one SPT and collapse completely. The simulations reveal the existence of similar collapses for the $(7,7),(9,9),(10,10),(11,11),(12,12)$ and $(13,13)$ SWCNT bundles. Taking the $(10,10) @(15,15)$
DWCNT bundle as an example, its collapse pressure $P_{d}=4.68 \mathrm{GPa}$ is found to be higher than either that of $(10,10)$ SWCNT bundle $\left(P_{d}=3 \mathrm{GPa}\right)$ or that of $(15$, 15) SWCNT bundle $\left(P_{d}=1.3 \mathrm{GPa}\right)$. This means that the outer tube acts as a protection shield, and the inner tube supports the outer one and increase its structure stability. The obtained results are consistent with the experimental results [53-55].

However, the response behaviors of the largerdiameter DWCNT bundles become complex, as shown in Fig. 5(c). The $(16,16) @(21,21)$ and $(19,19) @(24$, 24) DWCNT bundles still collapse after one SPT. Surprisingly, the $(15,15) @(20,20),(17,17) @(22,22),(18$, $18) @(23,23)$ and $(21,21) @(26,26)$ DWCNT bundles undergo two different types of SPT with their transition pressure $P_{c}$ and $P_{d}$ respectively, during which their cross sections change from deformed hexagon first to racetrack and then to peanut shape, and the DWCNT bundles collapse completely after the second SPT. It should be noted that the DWCNT bundles exhibit some small discontinuous volume change between the different racetrack-shaped cross sections. Two SPTs and the racetrack-shaped cross sections could be observed in future experiments if the pressure increment is selected properly.

Furthermore, it is interesting to ask why the racetrackshaped cross sections do not appear for the $(16,16) @$ $(21,21)$ and $(19,19) @(24,24)$ DWCNT bundles before their final collapse. The simulations reveal that the cross section symmetry of the outer tubes plays an important role in the collapse process. It is found that a necessary condition to form an ideal hexagonal lattice is that the outer tube itself has a $C_{6}$ or $C_{3}$ rotational axis, which matches to the hexagonal lattice symmetry. Thus, with increasing pressure, these outer tube's cross section can keep a better hexagon before the SPT. More importantly, these DWCNT bundles only undergo one SPT to reach the stable collapsed structure no matter how big the diameteris. Therefore, the symmetry of the outer tube is a very important factor to decide the cross section shape of the DWCNT under the pressure, and comparatively the effect of their inner tube is very small.

Structure transformations of zigzag@zigzag DWCNT hydrostatic pressure, which are found to show a qualitatively same response mechanism to external pressure with the armchair@armchair DWCNT bundles, are indicated in Table 1.

In order to have an insight into the relationship of the collapse pressure $P_{d}$ with the DWCNT diameter and its tube symmetry, Yang et al. [70] introduced the DWCNT's average radius $R_{\text {ave, }}$, which is defined as an average value of the outer and inner tube's radius of DWCNT. The variation of $P_{d}$ with $R_{\text {ave }}$ for the DWCNT bundles can be well fitted to $\sim 1 / R_{\text {ave }}^{3}$, as shown in Fig. 6 . The reason behind it is simply because the tube-tube 
coupling in a bundle is described by the van der Waals force, which has only a small effect on the bundle's collapse pressure. Both the SWCNT and DWCNT can be described by the continuum elasticity theory as the continuous hollow cylinders. Therefore, their bundles would have a similar response to the hydrostatic pressure, and the only difference is that the effective radius of DWCNT is the average of its inner and outer tubes' radius. On the other hand, it has been proved by the continuum elasticity theory [76] and the molecular dynamics simulations $[77,78]$ that the $P_{d}$ of an individual SWCNT is inversely proportional to its cubic radius. Thus, it is reasonable that the relation of $P_{d} \sim 1 / R_{\text {ave }}^{3}$ appears in the DWCNT bundle.

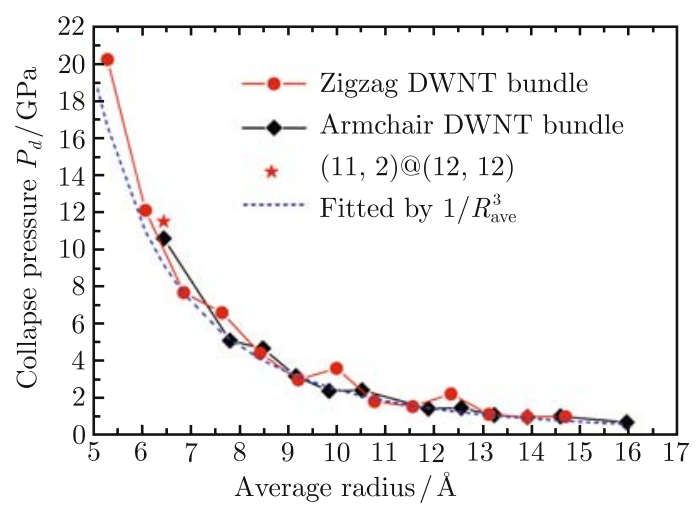

Fig. 6 Collapse pressure $P_{d}$ as a function of the average radius of DWCNT. $P_{d}$ can be well fitted to $\sim 1 / R_{\text {ave }}^{3}$. Adapted from Ref. [70] with permission.

In addition, for the DWCNT bundles, whose outer tube has $C_{6}$ or $C_{3}$ symmetry, the collapse pressure is found to be larger than the fitted result. This phenomenon is more obvious, especially for the zigzag@ zigzag DWCNT bundles, e.g., the $(9,0) @(18,0),(15$, $0) @(24,0),(21,0) @(30,0),(27,0) @(36,0)$ and $(33$, $0) @(42,0)$. The reason is that both of their outer and inner tubes have $C_{6}$ or $C_{3}$ symmetry, further enhancing the matching to the hexagonal lattice and increasing the structure stability of the system. This means the matching between the DWCNT symmetry and the lattice symmetry can increase the ability of the DWCNT bundle to resist the applied pressure.

\section{Raman modes of the deformed SWCNTs}

Recently, the mechanical deformation effect on the SWCNT's electronic properties has been successfully studied by using a simple $\pi$ electron tight-binding approximation [37, 38], and some interesting behaviors have been found, e.g., a symmetry breaking caused by the deformation lifts the degeneracy of energy levels. Most of the deformed SWCNTs show a metalsemiconductor transition, occurring repeatedly with increasing strain. Related experimental observations can be found in Refs. [32-38].

On the other hand, few investigations have been made on the the vibrational properties of the deformed SWCNTs. A systematical calculation on the vibrational modes and Raman spectra of the SWCNTs under a torsional or a uniaxial strain was made in Ref. [79], in which the Raman intensity is calculated using the eigenvectors of the vibrational modes, obtained by the first-principles method, and the polarizability parameters are obtained by the empirical bond polarizability model [11, 52]. It was found that almost all the predictions from the numerical calculation in Ref. [79] are consistent with the experimental observations [80-82].

4.1 Raman active modes of the SWCNTs under a uniaxial tensile strain

First we consider the Raman spectra of zigzag tubes. It is found that the low-energy modes almost do not change, but the high-energy ones would shift obviously under the tensile strain. And the intensities of the Raman active modes almost do not change at all. So, here, only their frequency shifts under the tensile strain are given in Fig. 7, where the tensile strain is defined as $e=\left(T / T_{0}\right)-1$. Here, $T$ and $T_{0}$ denote the lengths of the axial unit cells of the deformed and undeformed nanotubes, respectively.

It can be clearly seen from Fig. 7 that when the tensile strain strength increases:

(a) All the Raman modes shift linearly with the tensile strain strength.

(b) The deformation does not change the number of the Raman active vibration modes.

(c) The frequencies of the low-energy Raman active modes (below $1000 \mathrm{~cm}^{-1}$ ) almost do not change with the tensile strain strength.

(d) The frequencies of high-energy Raman active modes (above $1000 \mathrm{~cm}^{-1}$ ) decrease linearly with the tensile strain strength, showing two different decreasing slopes for: (i) $E_{1 g}$, and (ii) $E_{2 g}$ and $A_{1 g}$ modes. Also, the slopes seem to be insensitive to the tube radius.

The first behavior comes obviously from the very weak tensile strain used here, which makes the force constants change monotonically with the strain increasing. The other behaviors can be understood by the following reasons:

(i) The SWCNT's Poisson ratio $\sigma$ is equal to about 0.2 , which means the tube radius only decreases by about $1 \%$ even if the tube is elongated by $5 \%$. Also, the low-frequency phonon modes are mainly affected by the tube diameter, and so their Raman peak positions do not move much. Especially, the radial breathing mode (RBM), which is inversely proportional to the tube diameter, is found to do very small shifts (about $1 \mathrm{~cm}^{-1}$ ) 


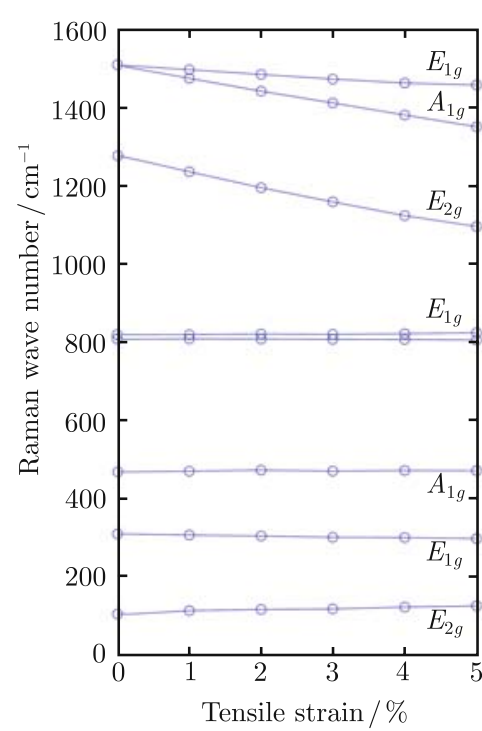

(a)

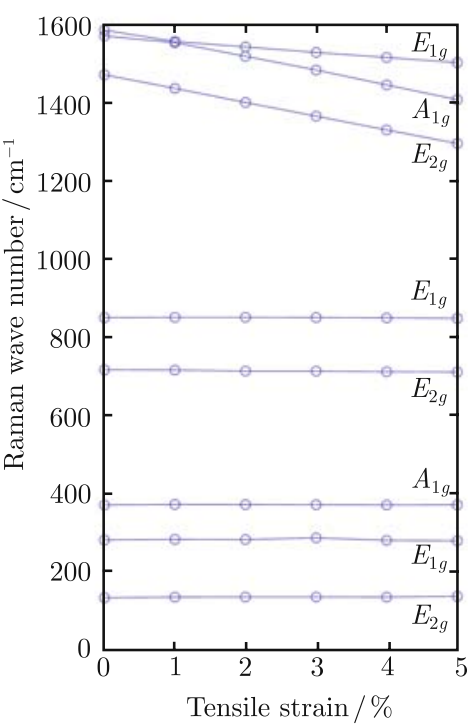

(b)

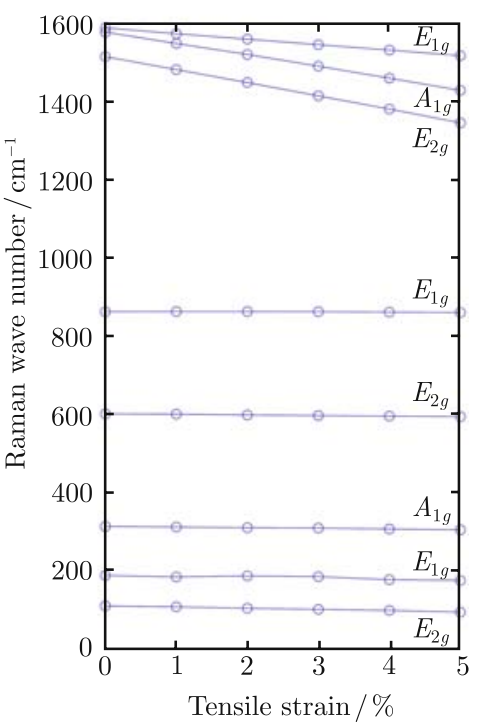

(c)

Fig. 7 Frequencies of the Raman active modes of the $(n, 0)$ zigzag tubes $(n=6,8$ and 10) under a tensile strain. Adapted from Ref. [79] with permission. (a) $(6,0) ;(\mathbf{b})(8,0) ;(\mathbf{c})(10,0)$.

when the tensile strain strength increases up to $5 \%$.

(ii) The uniaxial tensile strain does not change the SWCNT's symmetry, no matter whether the nanotube is chiral or achiral. According to the group theory, the number of nonresonant Raman active modes is only determined by the irreducible representations of the symmetric group, which also do not change under the tensile strain. Thus, the number of Raman active modes in the uniaxially deformed nanotubes should be exactly the same as that in the undeformed nanotubes.

(iii) The bonds parallel to the tube axis will be elongated when the tensile strain is applied. For the higherfrequency Raman modes, the vibrations are sensitive to the local bond structure, especially its length. When the bond length is elongated, the interaction between two nearby atoms will become weaker, leading to the smaller force constants, which makes the frequency of corresponding Raman mode become smaller.

The last characteristic in (d) has been found in the graphite system [83]. Following Ref. [83], we also calculated the Grüneisen tensor elements (GTEs):

$$
\kappa=-\frac{\mathrm{d} \ln \omega}{\mathrm{d} \ln (1+e)}
$$

where $\omega$ is the frequency of Raman active modes, and $e$ is the tensile strain. The GTEs vs the tube index $n$ of the zigzag tubs are plotted in Fig. 8, in which the same fitting function for the GTEs are also given, written as:

$$
\kappa=A+\frac{B}{n^{2}}+\frac{C}{n^{2}} \sin \left(\frac{2 \pi}{3} n+\phi_{0}\right)
$$

After making a simple least-squares fit, finally we can obtain $\kappa_{E_{1 g}}(r \rightarrow \infty)=0.897, \kappa_{A_{1 g}}(r \rightarrow \infty)=2.05$, and
$\kappa_{E_{2 g}}(r \rightarrow \infty)=1.95$, which can be checked by future experiments. This also offers a powerful experimental tool to determine the tensile strain strength, i.e., by detecting the Raman-active modes in the undeformed and deformed SWCNTs, one can easily estimate how big the applied tensile strain is.

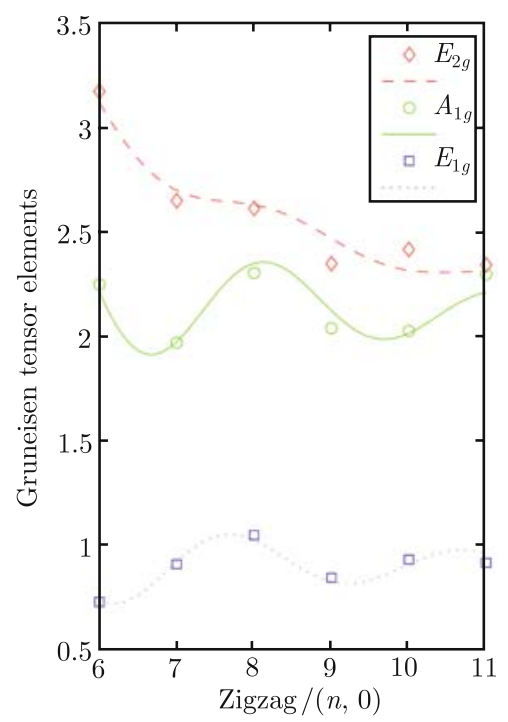

Fig. 8 The GTEs of the highest three Raman active modes for different size zigzag tubes $(n, 0),(n=6, \ldots, 11)$, which are obtained by first-principles calculations. The lines are fitting results (see text). Adapted from Ref. [79] with permission.

Now, we turn to other types of SWCNTs to study their Raman modes under a uniaxial strain. The frequencies of the Raman active modes for some armchair and chiral tubes under the tensile strains are shown in Fig. 9, from which one can find the same key characteristics as those in the Raman spectra of the zigzag tubes. The reason 


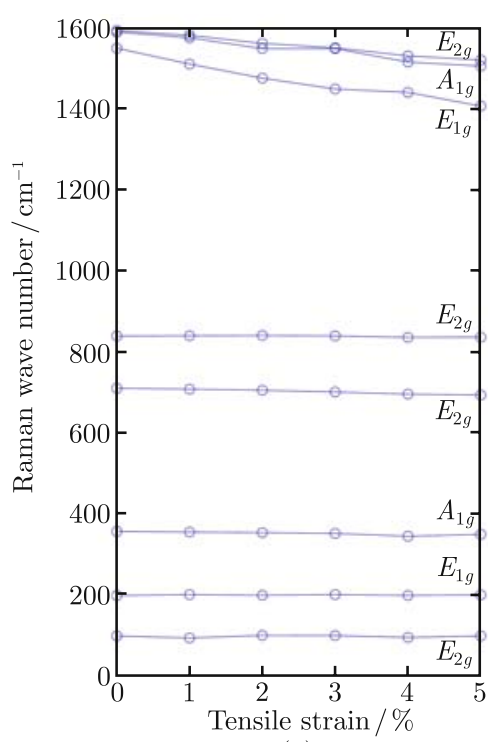

(a)

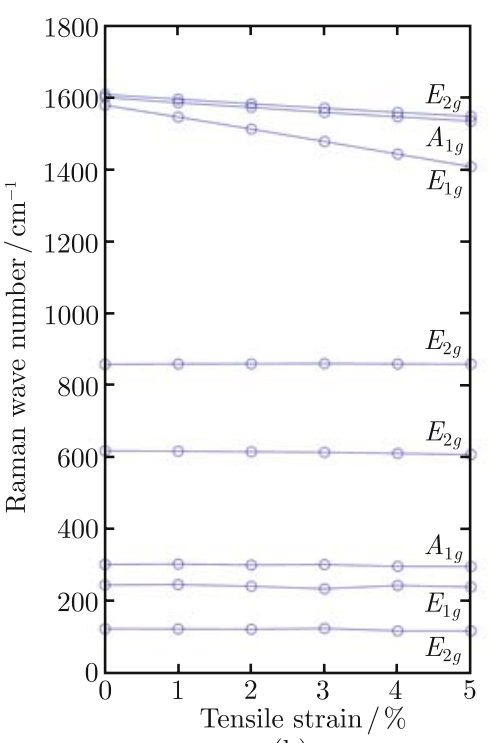

(b)

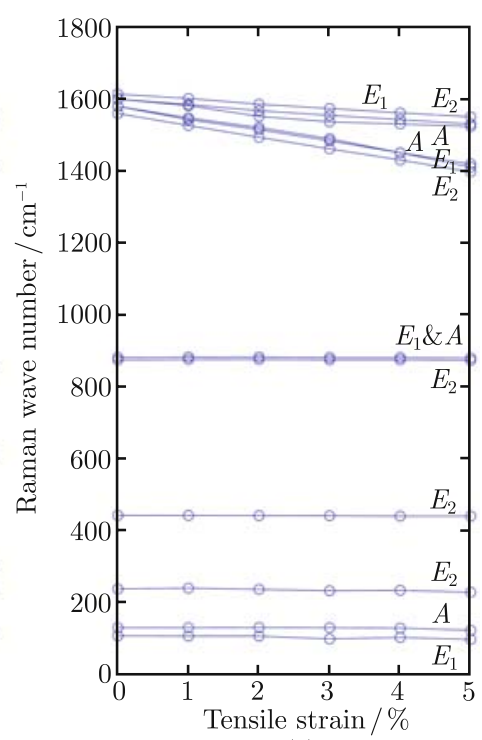

(c)

Fig. 9 Frequencies of the Raman active modes of some armchair and chiral SWCNTs under the tensile strains. (a) (5, 5), (b) $(6,6)$, (c) $(12,4)$. Adapted from Ref. [79] with permission.

should be the same as that for the zigzag tubes.

The GTEs of these tubes are also calculated. It is noted that the GTEs of the high-frequency Raman modes of armchair tubes can also be divided into two groups, and their values are very close to those of the zigzag tubes. For example, for the $(6,6)$ tube, $\kappa_{E_{1 g}}=$ $2.35, \kappa_{A_{1 g}}=0.861$, and $\kappa_{E_{2 g}}=0.794$. And for the (12, 4) tube, the GTEs of its six highest frequency Raman modes are: $2.25,2.27,2.28,0.994,0.907$ and 0.846 . So, it is very possible that there are only two kinds of the GTEs for the high-frequency Raman modes in any SWCNT. In fact, detailed analysis [79] shows that those modes with the larger GTEs are the tangential with atomic displacements parallel to the tube axis, and those modes with the smaller GTEs are the tangential with atomic displacements perpendicular to the tube axis.

4.2 Raman active modes of the SWCNTs under a torsional strain

We have also studied the torsional strain effect on the SWCNT's Raman modes. Here, we take three kinds of the SWCNTs with different chiralities, which are armchair $(5,5)$, zigzag $(10,0)$ and chiral $(12,4)$ tubes. The torsional angle $\gamma$ is defined in a similar way to that in Refs. [37, 38], i.e., it makes any point of $\left(r_{c}, r_{t}\right)$ on the 2-D projection plot of the tube surface to move to $\left(r_{c}-\tan \gamma r_{t}, r_{t}\right)$. After structure relaxation on both the lattice constant along the tube axis and the atomic positions, we can finally obtain a reasonable tube structure, which can be used further for the first principles calculations. Because of the symmetry, we only consider the case of positive $\gamma$ for the armchair and zigzag tubes.
The frequencies of Raman active modes for three different SWCNTs under the torsional strains are shown in Fig. 10. Also, the high-frequency part of the $(12,4)$ tube is presented in Fig. 11.

Compared with the tensile strain situation, we can find their several key characters:

(a) The low-frequency Raman active modes almost do not change.

(b) Some high-frequency modes are red-shifted and some others are blue-shifted.

(c) The Raman frequencies are not symmetric with respect to the positive and negative torsional strains.

(d) The number of Raman active modes changes under the torsional strain.

In fact, the first characteristic can be explained by the same reason as that under the tensile strain. For example, the radius of the optimized $(12,4)$ tube changes only about $0.2 \%$ when the torsional strain increases up to $6^{\circ}$.

The two bonds mirroring each other with a $\sigma_{v}$ symmetry will be elongated and shortened, respectively, under a torsional strain. As a result, some force constants decrease and some others increase under the torsional strain, thus making some Raman modes red-shifted, and some others blue-shifted.

The final characteristic should be ascribed to the symmetry breaking due to the torsional deformation. It is natural that the torsional strain will lower the symmetries of the SWCNTs, especially of the achiral SWCNTs, inducing some new Raman modes in the frequency range of about $400 \mathrm{~cm}^{-1}$ for the zigzag tube, which would be originally prohibited by the symmetry restriction of the undeformed SWCNTs.

In order to investigate the effect of torsional strain on 


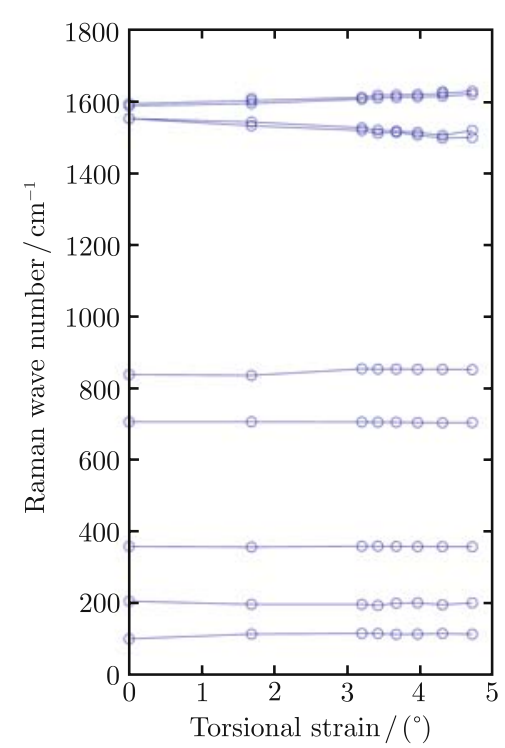

(a)

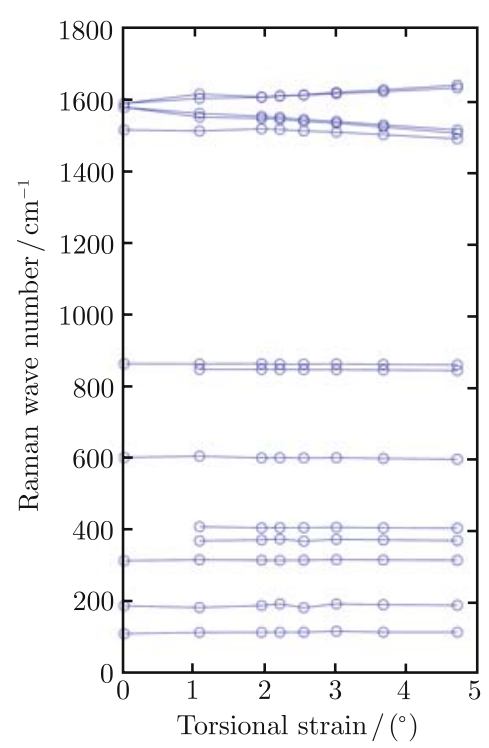

(b)

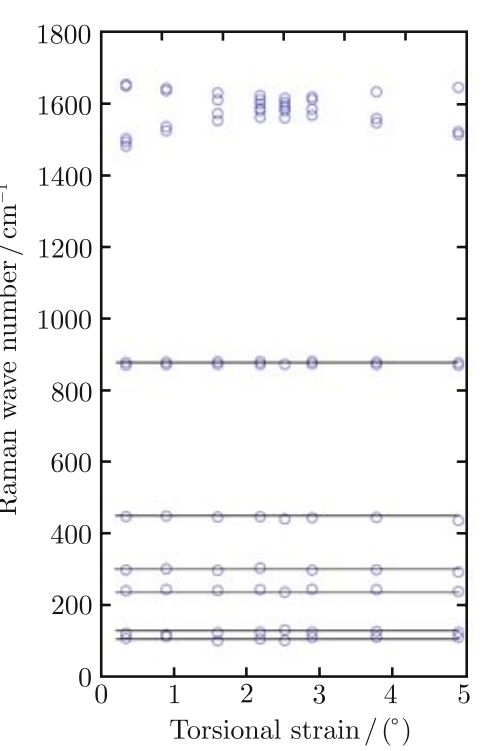

(c)

Fig. 10 Frequencies of Raman active modes vs. torsional strains for three SWCNTs. (a) $(5,5),($ b) $(10,0),(\mathbf{c})(12,4)$. Adapted from Ref. [79] with permission.

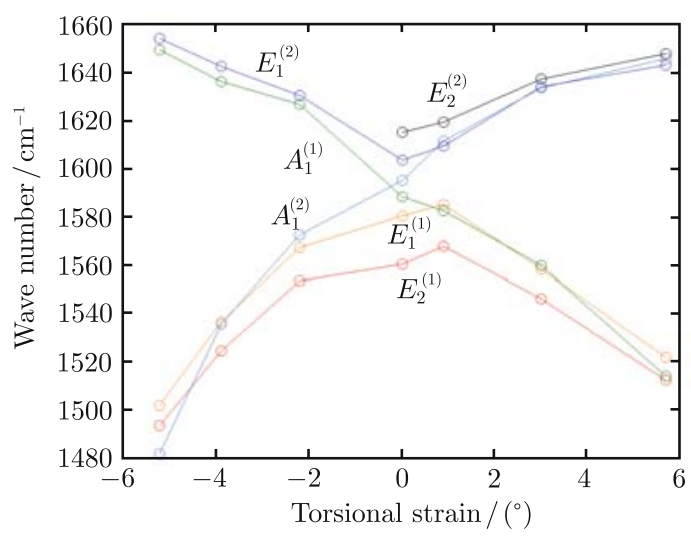

Fig. 11 Frequencies of high-frequency Raman active modes vs torsional strains for $(12,4)$ tube. Adapted from Ref. [79] with permission.

the high-frequency Raman modes, their GTEs are calculated, which are defined as:

$$
\kappa_{t}=-\frac{16}{3} \frac{\mathrm{d} \ln \omega}{\mathrm{d} \tan ^{2} \gamma}
$$

where $\omega$ is the frequency of Raman-active modes, and $\gamma$ is the torsional strain. This definition is obtained by assuming $\frac{\omega}{\omega_{0}}=\left(\frac{r}{r_{0}}\right)^{3 \kappa_{t}}$, which is taken from Ref. [83].

The $\kappa_{t}$ of the highest 4 Raman-active modes for the (5, 5) tube are found to be $-26.73,-26.52,19.85$ and 15.65 , respectively, and $\kappa_{t}$ of the highest 5 Raman-active modes for the $(10,0)$ tube are $-13.68,-30.68,-28.37,17.95$ and 19.80 , respectively. Because of the complexity of the high-frequency Raman modes of $(12,4)$ tube, here we only consider its two most intense modes lying at about $1600 \mathrm{~cm}^{-1}$, whose $\kappa_{t}$ are -32.29 and 14.03 , respectively. One can find that these values do not obviously relate to each other, which is different from the case under the tensile strain. Therefore, more investigations should be done to find the intrinsic mechanism to cause the shifts of the Raman-active modes under a torsional strain.

Finally, it can be found from Fig. 11 that the Raman frequencies are not symmetric for the positive and negative torsional strains for the $(12,4)$ tube. This is a natural result since the $(12,4)$ tube is a chiral SWCNT. So, if the future experiments are made to study the torsional deformation of the SWCNTs, it is valuable to pay more attention to the torsional direction.

\subsection{Radial-breathing-like mode of the collapsed}

SWCNT bundles under a hydrostatic pressure

Recently, the effect of hydrostatic pressure on the CNT bundle, especially its Raman spectra, has attracted much attention experimentally $[56,57,59,84-87]$ and theoretically $[16,88]$. Kahn et al. [16] studied the dependence of the Raman modes on the hydrostatic pressure for different nanotubes, and found that their shifts under the pressure differ from the corresponding ones of the graphite. It is found in Refs. $[56,57,59]$ that the RBM shifts towards higher wavenumbers with increasing hydrostatic pressure at a rate of $7 \sim 10 \mathrm{~cm}^{-1} \mathrm{GPa}^{-1}$. Refs. $[57,84]$ reported that before a structural transition at near $2 \mathrm{GPa}$, the tangential modes exhibited essentially the same pressure dependence of 5.7-5.8 and 5.3-6.1 $\mathrm{cm}^{-1} \mathrm{GPa}^{-1}$, respectively. The intensity and broadening of $R$ band decrease with increasing pressure and finally the RBM vanishes above a critical pressure [56-59], showing a structural phase transition (SPT) at this pressure. Meantime, the Raman spectra [56], in situ synchrotron X-ray diffraction 
Table 2 The frequencies of some characteristic Raman active modes and their pressure dependencies. The $\omega_{\text {expt }}$ and $\omega_{\text {theor }}$ denote the experimental and theoretical Raman frequencies of the structure at $0 \mathrm{GPa}$, respectively. Adapted from Ref. [89] with permission.

\begin{tabular}{|c|c|c|c|c|c|c|c|c|}
\hline \multirow{2}{*}{$\begin{array}{c}\text { Structure } \\
\text { region }\end{array}$} & \multicolumn{4}{|c|}{ RBM } & \multicolumn{4}{|c|}{$T$ band } \\
\hline & $\omega_{\text {expt }}$ & $\mathrm{d} \omega_{\operatorname{expt}} / \mathrm{d} p$ & $\omega_{\text {theor }}$ & $\mathrm{d} \omega_{\text {theor }} / \mathrm{d} p$ & $\omega_{\text {expt }}$ & $\mathrm{d} \omega_{\operatorname{expt}} / \mathrm{d} p$ & $\omega_{\text {theor }}$ & $\mathrm{d} \omega_{\text {theor }} / \mathrm{d} p$ \\
\hline Before & & & & & 1566 & 6.1 & 1583 & 9.3 \\
\hline \multirow[t]{2}{*}{$\mathrm{SPT}$} & 181 & 10.1 & 205 & 9.0 & 1571 & 11.0 & 1598 & 13.0 \\
\hline & & & & & 1591 & 10.1 & 1615 & 9.3 \\
\hline After & & & & & & 0.7 & & 1.6 \\
\hline \multirow[t]{2}{*}{$\mathrm{SPT}$} & & & & & & 5.9 & & 6.2 \\
\hline & & & & & & 5.8 & & 3.9 \\
\hline
\end{tabular}

data [60] and the optical absorption spectra [61] showed that the changes of both the geometrical structure and optical properties are reversible upon unloading the pressure. Sluiter and Kawazoe [62,63] used the continuum model to calculate the complete phase diagram of the CNT bundle under a hydrostatic pressure. Their results indicate that if considering stable states only, the tube's cross sections in a $(10,10)$ SWCNT bundle round up to 1.9 GPa, and become racetrack-shaped between 1.9 and 6.0 GPa. More details about this hydrostatic pressure effect on the Raman modes can be found in the review article [88].

We carried out a series of numerical calculations to study the hydrostatic pressure effect on the Raman active modes of the $(10,10)$ SWCNT bundle [89]. Significantly, an RBM-like mode appears at about $509 \mathrm{~cm}^{-1}$, which could be considered as a fingerprint of the SPT happened in the SWCNT bundle. Thus the RBM-like mode can be further used to determine experimentally the microscopic structure of the SWCNT bundle after the SPT.

In all the structures, the Raman frequencies were found to increase with increasing external pressure. The pressure dependence are summarized in Table 2 , in which the experimental data taken from Ref. [57] are also presented. From Table 2, we can find that although the first-principles calculation overestimates the Raman frequency systematically, it can reproduce most of the experimental dependence of the Raman frequency on the hydrostatic pressure. Therefore, we can use the firstprinciples results to further investigate in detail the vibrational modes of the $(10,10)$ bundle under hydrostatic pressure.

The optimized structures of a $(10,10)$ SWCNT bundle under different hydrostatic pressures are shown in Fig. 12. As the pressure increases, the tube's surface deformation energy increases, and the intertube distance decreases, increasing the van der Waals (vdW) energy. When the pressure increases up to a critical value, it becomes energetically favorable to reduce the tube's inner volume rather than simply reduce the intertube distance, thus making the system undergo an SPT, and the tube spontaneously collapses to form a peanut-like cross- section, which is consistent with the recent molecular dynamics simulations [64] and continuum model analysis [62, 63]. Such a kind of structural change should exhibit some important characteristics in the vibrational properties of the SWCNT bundles.

The rotational mode (A), and the RBM or RBMlike mode (B) of the SWCNT bundle at $0.45,2.8$ and $5 \mathrm{GPa}$ are given in Fig. 12(a)-(c), respectively. First, in Fig. 12(a), one may notice that the tube's crosssection is not a perfect circle. This is because the rotational symmetry of the $(10,10)$ tube is incompatible with the hexagonal lattice symmetry in the corresponding bundle, making the tube's cross-section deformed slightly. Second, the atomic motions in the rotational mode (A) before and after the SPT are always almost along the tube surface, but its frequency cannot remain zero because of the non-circular crosssection and the tube-tube interaction. This mode is IR active, making it observed possibly in the IR spectra. In addition, because of the hexagonal symmetry of
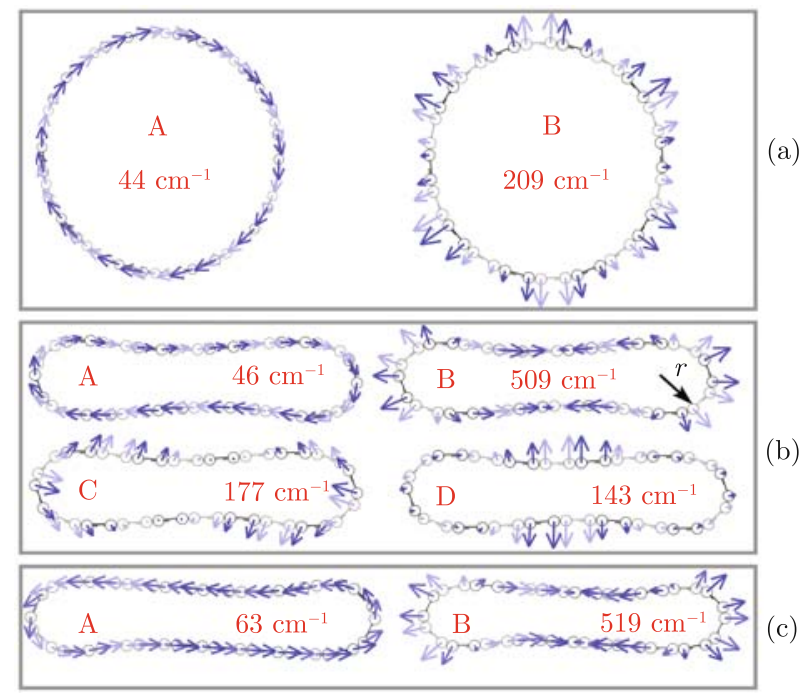

Fig. 12 Some characteristic vibrational modes of the SWCNT bundle: (a) at $0.45 \mathrm{GPa}$ before the SPT, (b) at $2.8 \mathrm{GPa}$ after the SPT, and (c) at $5 \mathrm{GPa}$. The small circles represent the carbon atoms and the straight lines between them indicate the bonds. The arrows represent the atomic motions. Deeper color arrow means the corresponding atom is nearer to the reader. $r$ is the radius of its two circular ends. Adapted from Ref. [89] with permission. 
the unit cell in the bundle, the atomic motions in the RBM are not the same for every atom, as seen from Fig. 12(a), which exhibit approximately the hexagonal symmetry too, indicating that the RBM frequency in the SWCNT bundle will not be the same as that in the isolated SWCNT.

Special attention should be paid to the mode (B) of $509 \mathrm{~cm}^{-1}$ at $2.8 \mathrm{GPa}$ after the SPT in Fig. 12(b). In this mode, most of the atomic motions on the two ends are perpendicular to the tube surface, showing in-phase vibrations, but the atoms' motions in the flat region are along the tube surface. This mode also exists at $5 \mathrm{GPa}$, as shown in Fig. 12(c), which can be regarded as an RBM-like mode appearing only after the SPT of the bundle. In fact, a previous researcher also found a similar mode in a capped SWCNT [90], which comes from a mixing of the RBM of the $\mathrm{C}_{60}$ hemisphere and the tangential mode of capped SWCNT. Because most of the atomic motions in the mode are in-phase, this RBM-like mode has a large Raman intensity, as shown in Fig. 13, making it observable in the experimental Raman spectra of the bundle after the SPT.

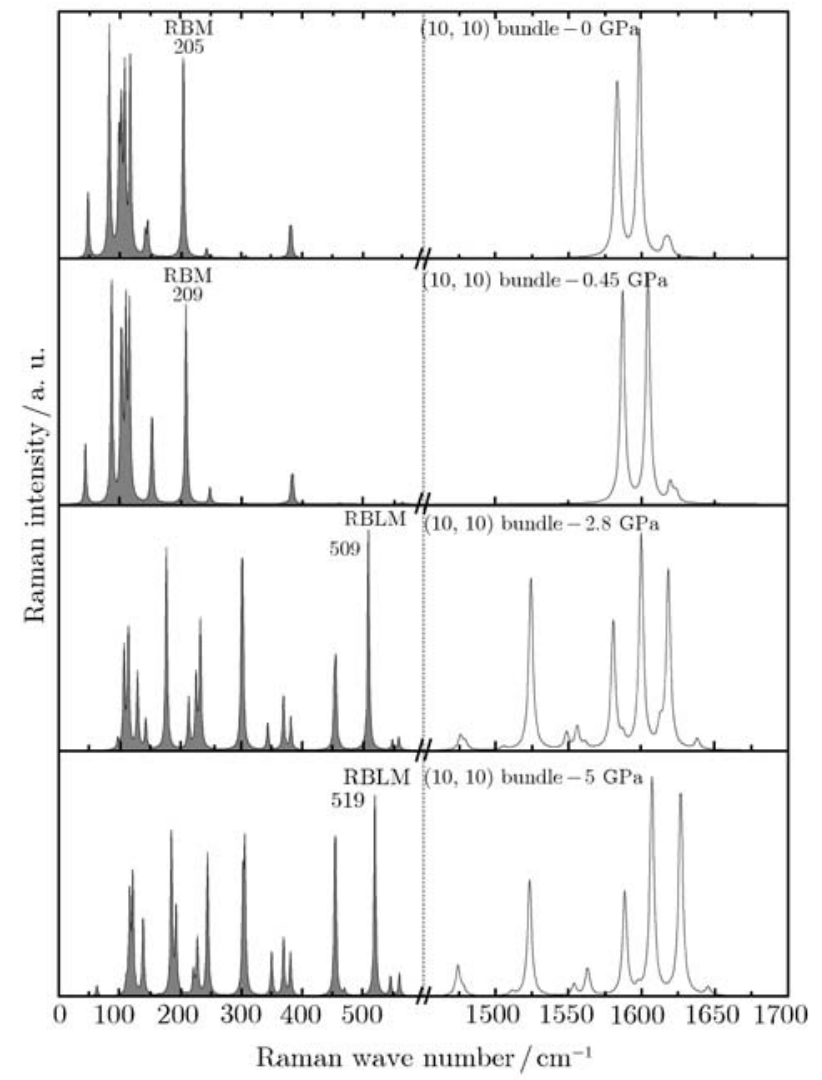

Fig. 13 The nonresonant Raman spectra of $(10,10)$ bundle at different pressures. From bottom to top, the external pressure is $0,0.45,2.8$ and $5 \mathrm{GPa}$, respectively. The frequencies of the RBM and RBM-like modes are labeled. Adapted from Ref. [89] with permission.

It is well known that the RBM frequency is inversely proportional to tube diameter [see Eq. (1)]. To exam- ine further the RBM-like mode in Fig. 12(b), we have calculated the radius of the circular end in Fig. 12(b), which is found to be $r=\frac{d_{t}}{2} \approx 2.26 \AA$. After taking $D=$ $228 \mathrm{~nm} / \mathrm{cm}^{-1}$ from Ref. [57], which was also obtained by the first-principles method, one can obtain $f_{\mathrm{RBM}}=504.4$ $\mathrm{cm}^{-1}$. This value is very close to our first-principles result of $509 \mathrm{~cm}^{-1}$, and a difference of only several $\mathrm{cm}^{-1}$ comes from the tube-tube interactions. In order to make a more accurate comparison between them, we directly take out one isolated tube from the collapsed bundle at $2.8 \mathrm{GPa}$, and then calculate the frequency of its RBMlike mode, which is found to be $501 \mathrm{~cm}^{-1}$. Thus, a very good agreement between the normal mode frequency of an isolated tube and the RBM of an equivalent SWCNT composed of the circular ends of a collapsed tube in the bundle has been obtained, indicating that the normal mode (B) in Fig. 12(b) can indeed be considered as an RBM-like mode of the tube bundle after the SPT. It is valuable to mention that the appearance of this RBMlike mode indicates the existence of the two circular ends for the tubes in the bundle after the SPT, which furthermore can be regarded as a fingerprint of the SPT. More importantly, the frequency of this RBM-like mode is only determined by the radii of the two circular ends, and so is mainly influenced by the external pressure, but not the radius of the original tube. As shown in Fig. 12(c), when external pressure increases to $5 \mathrm{GPa}$, the frequency of the RBM-like mode is changed to $519 \mathrm{~cm}^{-1}$. So this mode can also be used to determine experimentally the microscopic structure of the bundle after the SPT, no matter what kind of SWCNT bundle is used originally.

Another interesting Raman-active normal mode (C) in Fig. 12(b) is a quadrupole vibration mode, which often has a strong intensity in the Raman spectra of clusters. Also, the lowest $E_{2 g}$ Raman active mode of the SWCNT is also a quadrupole mode. The similarity between these modes may imply that the mode $(\mathrm{C})$ originates from the lowest $E_{2 g}$ Raman active mode of the SWCNT. In other words, the $E_{2 g}$-like modes of the two circular ends would combine together to give the mode (C) in Fig. 12(b), which could be understood by the following argument. It is known that the frequency of the SWCNT's lowest $E_{2 g}$ Raman active mode is inversely proportional to its squared diameter, i.e., $f \propto d_{t}^{-2}$ [12]. Taking the radius of the circular ends to be $r=\frac{d_{t}}{2} \approx 2.26 \AA$, its $E_{2 g}$ mode frequency will become $f \approx\left(\frac{6.8}{2.26}\right)^{2} \times 17 \approx 154 \mathrm{~cm}^{-1}$, where the radius of $(10,10)$ tube is equal to $r_{(10,10)} \approx$ $6.8 \AA$, and $17 \mathrm{~cm}^{-1}$ is the $E_{2 g}$ mode frequency of the isolated $(10,10)$ SWCNT. After considering the up-shift of the frequency induced by the intertube interaction, this value is compatible with the mode frequency of 177 $\mathrm{cm}^{-1}$, given in Fig. 12(b). So, this mode could also be 
observed in future Raman experiments.

Next, the mode (D) of $143 \mathrm{~cm}^{-1}$ in Fig. 12(b) is mainly contributed by the out-phase motions of the nearby flat walls, which can be regarded as the $B_{2 g}$ mode of the graphite with its frequency of $127 \mathrm{~cm}^{-1}$. But now due to pressure effect, its frequency increases to $143 \mathrm{~cm}^{-1}$. When pressure increases up to $5 \mathrm{GPa}$, the frequency of this mode further increases to $193 \mathrm{~cm}^{-1}$.

\section{Conclusions}

The structural and vibrational properties of the SWCNT bundles and DWCNT ones under the hydrostatic pressure have been investigated by the first principles method. It is found that there exist three different collapsed structures for the SWCNT bundle, i.e., the parallel, herringbone and in-between structures, showing different electronic, optical and vibrational properties their, by which an efficient experimental method is proposed to distinguish unambiguously the three different collapsed structures. The structural transformations of the DWCNT bundles under the hydrostatic pressure are found to be different from those of the SWCNT bundles and the isolate DWCNT. The DWCNT bundles can exhibit one or two structural phase transitions (SPTs), depending on their symmetry and tube's diameter in the bundle.

The nonresonant Raman spectra of the SWCNTs under uniaxial and torsional strains are systematically studied using the first-principles calculations and the EBPM. It is found that the frequencies and the intensities of the low-frequency Raman-active modes almost do not change under the uniaxial tensile strain, while their highfrequency part shifts downward linearly with two different slopes for any kind of SWCNTs. By investigating the Grüneisen tensor elements (GTEs), it is shown that there is a common feature for the Raman shift of all kinds of the SWCNTs. Moreover, a universal formula of the GTEs is suggested. On the other hand, it is found that although the frequencies and the intensities of the low-frequency Raman-active modes still change very little under the torsional strain, some of the high-frequency modes are red-shifted and some others blue-shifted. The reasons behind all these phenomena have been discussed. Finally, it is found that the shifts of the Raman frequencies of the chiral SWCNTs are not symmetric under the positive and negative torsional strains. Therefore, it is valuable to pay more attention to the torsional direction in future experiments.

The vibrational modes of the $(10,10)$ SWCNT bundle under the hydrostatic pressure are studied in detail by using the first-principles calculations. It is found that its vibrational modes, especially after the SPT, are very different from those without the applied pressure. Sev- eral important results are obtained, e.g., disappearance of the original RBM, and the appearance of an RBMlike mode and a quadrupole vibrational mode after the SPT, which can be considered as a fingerprint of the happened SPT, and used to measure accurately the microscopic structure of the SWCNT bundle after the SPT in future experiment.

Finally, we should mention that it would be very interesting to determine experimentally the structural and vibrational characteristics of the deformed CNTs and CNT bundles, found above theoretically.

Acknowledgements The authors acknowledge the support from the National Natural Science Foundation of China (grant No. 10474035), and also from a grant for the State Key Program of China (Grant No. 2004CB619004).

\section{References}

1. S. Iijima, Nature (London), 1991, 354: 56

2. T. W. Ebbesen and P. M. Ajayan, Nature (London), 1992, 358: 220

3. J. Kong, H. T. Soh, A. M. Cassell, C. F. Quate, and H. Dai, Nature (London), 1998, 395: 878

4. R. Saito, G. Dresselhaus, and M. S. Dresselhaus, Physical Properties of Carbon Nanotubes, London: Imperial College Press, 1998

5. M. S. Dresselhaus, G. Dresselhaus, and Ph. Avouris, Carbon Nanotubes: Synthesis, Structure, Properties and Applications, Vol. 80 of Springer Series in Topics in Applied Physics, Berlin: Springer-Verlag, 2001

6. M. S. Dresselhaus and P. C. Eklund, Adv. Phys., 2000, 49: 705

7. A. M. Rao, E. Richter, S. Bandow, B. Chase, P. C. Eklund, K. A. Williams, S. Fang, K. R. Subbaswamy, M. Menon, A. Thess, R. E. Smalley, G. Dresselhaus, and M. S. Dresselhaus, Science, 1997, 275: 187

8. A. Kasuya, Y. Sasaki, Y. Saito, K. Tohji, and Y. Nishina, Phys. Rev. Lett., 1997, 78: 4434

9. J. Hone, B. Batlogg, Z. Benes, A. T. Johnson, and J. E. Fischer, Science, 2000, 289: 1730

10. R. A. Jishi, L. Venkataraman, M. S. Dresselhaus, and G. Dresselhaus, Chem. Phys. Lett., 1993, 209: 77

11. R. Saito, T. Takeya, T. Kimura, G. Dresselhaus, and M. S. Dresselhaus, Phys. Rev. B, 1998, 57: 4145

12. R. Saito, T. Takeya, T. Kimura, G. Dresselhaus, and M. S. Dresselhaus, Phys. Rev. B, 1999, 59: 2388

13. V. P. Sokhan, D. Nicholson, and N. Quirke, J. Chem. Phys., 2000, 113: 2007

14. V. N. Popov, V. E. Van Doren, and M. Balkanski, Phys. Rev. B, 1999, 59: 8355

15. V. N. Popov, V. E. Van Doren, and M. Balkanski, Phys. Rev. B, 2000, 61: 3078

16. D. Kahn and J. P. Lu, Phys. Rev. B, 1999, 60: 6535

17. D. Kahn, K. W. Kim, and M. A. Strosico, J. Appl. Phys., 2001, 89: 5107

18. M. Xia, S. Zhang, S. Zhao, and E. Zhang, Physica B, 2004, 
344: 66

19. G. D. Mahan, Phys. Rev. B, 2002, 65: 235402

20. S. Zhang, M. Xia, S. Zhao, T. Xu, and E. Zhang, Phys. Rev. B, 2003, 68: 075415

21. C. Journet, W. K. Maser, P. Brenier, A. Loiseau, M. Lamy de la Chapelle, S. Lefrant, P. Deniard, R. Lee, and J. E. Fischer, Nature (London), 1997, 388: 756

22. J. M. Holden, P. Zhou, X. X. Bi, P. C. Eklund, S. Bandow, R. A. Jishi, K. Das Chowdhury, G. Dresselhaus, and M. S. Dresselhaus, Chem. Phys. Lett., 1994, 220: 186

23. A. G. SouzaFilho, S. G. Chou, G. G. Samsonidze, G. Dresselhaus, M. S. Dresselhaus, L. An, J. Liu, A. K. Swan, M. S. Unlu, B. B. Goldberg, A. Jorio, A. Gruneis, and R. Saito, Phys. Rev. B, 2004, 69: 115428

24. H. J. Dai, A. G. Rinzler, P. Nikolaev, A. Thess, D. T. Colbert, and R. E. Smalley, Chem. Phys. Lett., 1996, 260: 471

25. R. Saito, R. Matsuo, T. Kimura, G. Dresselhaus, and M. S. Dresselhaus, Chem. Phys. Lett., 2001, 348: 187

26. K. Tanaka, H. Aoki, H. Ago, T. Yamabe, and K. Okahara, Carbon, 1997, 35: 121

27. Y. K. Kwon and D. Tomanek, Phys. Rev. B, 1998, 58: R16001

28. M. B. Nardelli, C. Brabec, A. Maiti, C. Roland, and J. Bernholc, Phys. Rev. Lett., 1998, 80: 313

29. T. Hertel, R. Martel, and Ph. Avouris, J. Phys. Chem. B, 1998, 102: 910

30. T. Hertel, R. E. Walkup, and Ph. Avouris, Phys. Rev. B, 1998, 58: 13870

31. Yu. N. Gartstein, A. A. Zakhidov, and R. H. Baughman, Phys. Rev. B, 2003, 68: 15415

32. W. T. Thomas, C. W. Zhou, J. Kong, and H. J. Dai, Appl. Phys. Lett., 2000, 76: 2414

33. W. Clauss, D. J. Bergeron, and A. T. Johnson, Phys. Rev. B, 1998, 58: R4266

34. R. Heyd, A. Charlier, and E. McRae, Phys. Rev. B, 1997, 55: 6820

35. D. W. Brenner, J. D. Schall, J. P. Mewlkill, D. A. Shenderova, and S. B. Sinnott, J. Br. Interplanet. Soc., 1998, 51: 137

36. C. L. Kane and E. J. Mele, Phys. Rev. Lett., 1997, 78: 1932

37. L. Yang, M. P. Anantram, J. Han, and J. P. Lu, Phys. Rev. B, 1999, 60: 13874

38. L. Yang and J. Han, Phys. Rev. Lett., 2000, 85: 154

39. H. Jiang, G. Wu, X. P. Yang, and J. M. Dong, Phys. Rev. B, 2004, 70: 125404

40. I. Božović, M. Vujičić, and F. Herbut, J. Phys. A, 1978, 11: 2133

41. I. Božović and M. Vujičić, J. Phys. A, 1981, 14: 777

42. M. Damnjanović, Phys. Lett. A, 1983, 94: 337

43. N. Božović, I. Božović, and M. Damnjanović, J. Phys. A: Math. Gen., 1985, 18: 923

44. N. Božović, I. Božović, and M. Damnjanović, J. Phys. A: Math. Gen., 1984, 17: 747

45. M. Damnjanović, I. Milošević, T. Vuković, and R. Sredanović, Phys. Rev. B, 1999, 60: 2728

46. Ofir E. Alon, Phys. Rev. B, 2001, 63: 201403(R)
47. L. H. Ye, B. G. Liu, D. S. Wang, and R. Han, Phys. Rev. B, 2004, 69: 235409

48. K. Parlinski, Z. Q. Li, and Y. Kawazoe, Phys. Rev. Lett., 1997, 78: 4063

49. G. Viliani, R. DellAnna, O. Pilla, M. Montagna, G. Ruocco, G. Signorelli, and V. Mazzacurati, Phys. Rev. B, 1995, 52: 3346

50. M. Lazzeri and F. Mauri, Phys. Rev. Lett., 2003, 90: 036401

51. S. Sanguinetti, G. Benedek, M. Righetti, and G. Onida, Phys. Rev. B, 1994, 50: 6743

52. S. Guha, J. Menendez, J. B. Page, and G. B. Adams, Phys. Rev. B, 1996, 53: 13106

53. P. Puech, H. Hubel, D. J. Dunstan, R.R. Bacsa, C. Laurent, and W. S. Bacsa, Phys. Rev. Lett., 2004, 93: 095506

54. J. Arvanitidis, D. Christofilos, K. Papagelis, K. S. Andrikopoulos, T. Takenobu, Y. Iwasa, H. Kataura, S. Ves, and G. A. Kourouklis, Phys. Rev. B, 2005, 71: 125404

55. J. Arvanitidis, D. Christofilos, K. Papagelis, T. Takenobu, Y. Iwasa, H. Kataura, S. Ves, and G. A. Kourouklis, Phys Rev. B, 2005, 72: 193411

56. U. D. Venkateswaran, A. M. Rao, E. Richter, M. Menon, A. Rinzler, R. E. Smalley, and P. C. Eklund, Phys. Rev. B, 1999, 59: 10928

57. M. J. Peters, L. E. McNeil, J. P. Lu, and D. Kahn, Phys. Rev. B, 2000, 61: 5939

58. C. Thomsen, S. Reich, A. R. Goni, H. Jantoljak, P. M. Rafailov, I. Loa, K. Syassen, C. Journet, and P. Bernier, Phys. Status Solidi B, 1999, 215: 435

59. C. Thomsen, S. Reich, H. Jantoljak, I. Loa, K. Syassen, M. Burghard, G. S. Duesberg, and S. Roth, Appl. Phys. A, 1999, 69: 309

60. J. Tang, L. C. Qin, T. Sasaki, M. Yudasaka, A. Matsushita, and S. Iijima, Phys. Rev. Lett., 2000, 85: 1887

61. S. Kazaoui, N. Minami, H. Yamawaki, K. Aoki, H. Kataura, and Y. Achiba, Phys. Rev. B, 2000, 62: 1643

62. M. H. F. Sluiter, V. Kumar, and Y. Kawazoe, Phys. Rev. B, 2002, 65: 161402

63. M. H. F. Sluiter and Y. Kawazoe, Phys. Rev. B, 2004, 69: 224111

64. J. A. Elliott, J. K. W. Sandler, A. H. Windle, R. J. Young, and M. S. P. Shaffer, Phys. Rev. Lett., 2004, 92: 095501

65. X. H. Zhang, Z. F. Liu, and X. G. Gong, Phys. Rev. Lett., 2004, 93: 149601

66. S. P. Chan, W. L. Yim, X. G. Gong, and Z. F. Liu, Phys. Rev. B, 2003, 68: 075404

67. X. H. Zhang, D. Y. Sun, Z. F. Liu, and X. G. Gong, Phys. Rev. B, 2004, 70: 035422

68. V. Gadagkar, P. K. Maiti, Y. Lansac, A. Jagota, and A. K. Sood, Phys. Rev. B, 2006, 73: 085402

69. X. P. Yang, G. Wu, J. Zhou, and J. M. Dong, Phys. Rev. B, 2006, 73: 235403

70. X. P. Yang, G. Wu, J. Zhou, and J. M. Dong, Appl. Phys. Lett., 2006, 89: 113101

71. X. P. Yang and G. Wu, Europhys. Lett., 2008, 81: 47003

72. P. Delaney, H. J. Choi, J. Ihm, S. G. Louie, and M. L. Cohen, Nature, 1998, 391: 466

73. P. Delaney, H. J. Choi, J. Ihm, S. G. Louie, and M. L. 
Cohen, Phys. Rev. B, 1999, 60: 7899

74. M. Ouyang, J. L. Huang, C. L. Cheung, and C. M. Lieber, Science, 2001, 292: 702

75. G. Y. Guo, K. C. Chu, D. S. Wang, and C. G. Duan, Phys. Rev. B, 1996, 69: 205416

76. B. I. Yakobson, C. J. Brabec, and J. Bernholc, Phys. Rev. Lett., 1996, 76: 2511

77. R. B. Capaz, C. D. Spataru, P. Tangney, M. L. Cohen, and S. G. Louie, Phys. Status Solidi B, 2004, 241: 3352

78. P. Tangney, R. B. Capaz, C. D. Spataru, M. L. Cohen, and S. G. Louie, Nano Lett., 2005, 5: 2268

79. G. Wu, J. Zhou, and J. M. Dong, Phys. Rev. B, 2005,72: 115411

80. S. B. Cronin, A. K. Swan, M. S. Ünlü, B. B. Goldberg, M. S. Dresselhaus, and M. Tinkham, Phys. Rev. Lett., 2004, 93: 167401

81. S. B. Cronin, A. K. Swan, M. S. Ünlü, B. B. Goldberg, M. S. Dresselhaus, and M. Tinkham, Phys. Rev. B, 2005, 72: 035425
82. X. Duan, H. Son, B. Gao, J. Zhang, T. Wu, G. G. Samsonidze, M. S. Dresselhaus, Z. Liu, and J. Kong, Nano Lett., 2007, 7: 2116

83. M. Hanfland, H. Beister, and K. Syassen, Phys. Rev. B, 1989, 39: 12598

84. S. Reich, H. Jantoljak, and C. Thomsen, Phys. Rev. B, 2000, 61: R13389

85. J. Sandler, M. S. P. Shaffer, A. H. Windle, M. A. MontesMoran, C. A. Cooper, R. J. Young, and M. P. Halsall, Phys. Rev. B, 2003, 67: 035417

86. C. A. Cooper, R. J. Young, and M. Halsal, Composites, Part A, 2001, 32A: 401

87. M. Lucas and R. J. Young, Phys. Rev. B, 2004, 69: 085405

88. I. Loa, J. Raman Spectrosc., 2003, 34: 611.

89. G. Wu, X. P. Yang, and J. M. Dong, Appl. Phys. Lett., 2006, 88: 223114

90. L. Vitali, M. Burghard, M. A. Schneider, L. Liu, S. Y. Wu, C. S. Jayanthi, and K. Kern, Phys. Rev. Lett., 2004, 93: 136103 